

\title{
COMPUTATIONAL MODELS OF MATERIAL INTERFACES FOR THE STUDY OF EXTRACORPOREAL SHOCK WAVE THERAPY
}

\author{
Kirsten Fagnan, Randall J. LeVeque and Thomas J. Matula
}

\begin{abstract}
Extracorporeal shock wave therapy (ESWT) is a noninvasive treatment for a variety of musculoskeletal ailments. A shock wave is generated in water and then focused using an acoustic lens or reflector so the energy of the wave is concentrated in a small treatment region where mechanical stimulation in principle enhances healing. In this work we have computationally investigated shock wave propagation in ESWT by solving a Lagrangian form of the isentropic Euler equations in the fluid and linear elasticity in the bone using high-resolution finite volume methods. We solve a full three-dimensional system of equations and use adaptive mesh refinement to concentrate grid cells near the propagating shock. We can model complex bone geometries, the reflection and mode conversion at interfaces, and the propagation of the resulting shear stresses generated within the bone. We discuss the validity of our simplified model and present results validating this approach.
\end{abstract}

\section{Introduction}

Extracorporeal shock wave therapy (ESWT) is a noninvasive treatment for musculoskeletal conditions such as bone fractures that fail to heal (nonunions), necrotic wounds, and strained tendons [55; 40]. In this treatment a shock wave is generated in water and then focused using an acoustic lens or reflector so that the energy of the wave is concentrated in a small treatment region. This technique has been used since the 1980's, more widely in Europe and Asia than in the US, where it is still considered experimental and has limited FDA approval.

Although the underlying biological mechanisms are not well understood [42], the mechanical compressional and/or shear stress caused by the propagating shock wave is thought to stimulate healing $[42 ; 54 ; 26 ; 39 ; 53 ; 43 ; 44 ; 30 ; 13 ; 27 ; 11$; 23]. In addition to stress, a number of other biological mechanisms potentially play a role in the body's response to ESWT. The focus of this study, however, is on mechanical stress deposition and computational tools for studying this phenomenon.

MSC2010: 92-08, 92C50, 65M08.

Keywords: high-resolution finite volume methods, computational biology, shock wave therapy. 
Computational models for shock wave propagation and focusing can aid in the study of ESWT. In particular, there are many open questions concerning the interaction of shock waves with complex three-dimensional geometries such as bone embedded in tissue. In this paper we present a new method for studying ESWT that incorporates the fluid and solid materials in a set of coupled, nonlinear partial differential equations that are solved using high-resolution finite volume methods. In order to model the wave interaction with complex three-dimensional geometries, we employed adaptive mesh refinement to concentrate the finest grid around then propagating shock wave.

Because of the difference in material properties, a wave hitting the tissue/bone interface will be partially reflected, and the transmitted wave will have a modified strength and direction of propagation. This can greatly affect the location and size of the focal region and the peak pressure amplitude. Also, although the shock wave is primarily a pressure wave in soft tissue (which has a very small shear modulus), at a bone interface mode conversion takes place and shear waves as well as compressional waves are transmitted into the bone, generating a dynamically applied load.

The medical shock wave devices are similar to those used for extracorporeal shock wave lithotripsy (ESWL), a widely used nonsurgical treatment for kidney stones in which the focused shock waves have sufficient amplitude to pulverize the kidney stone. In shock wave therapy the amplitudes are generally smaller and the goal is mechanical stimulation rather than destruction, although in some applications such as the treatment of heterotopic ossifications (HO) (see Section 5.4) larger amplitudes may be used.

Figure 1 shows the geometry of a laboratory shock wave device modeled on the
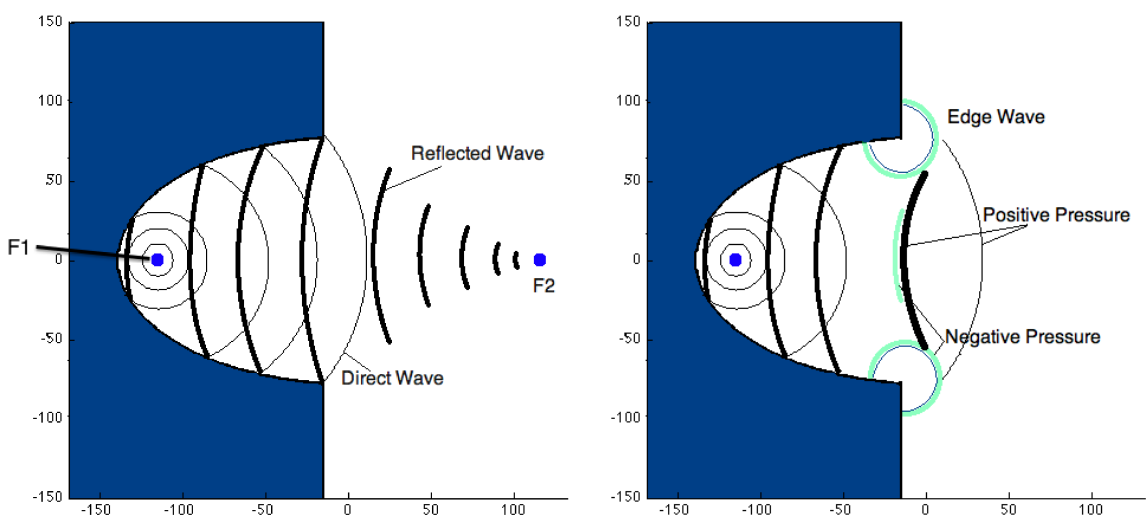

Figure 1. Cartoon of the Dornier HM3 Lithotripter. Left: the spherical wave is generated at F1, reflects off the ellipsoid and the reflected wave focuses at F2. Right: the creation of the edge waves at the corner of the ellipsoid and the contribution of negative pressure to the tail of the ESWT pressure wave. 
clinical Dornier HM3 lithotripter. The three-dimensional axisymmetric geometry consists of an ellipsoidal reflector made out of metal and a cavity filled with water. A spark plug at the focus of the ellipse marked F1 generates a bubble which collapses and creates a spherical shock wave that reflects and focuses at F2. The major and minor axes of the ellipsoid in the HM3 are $a=140 \mathrm{~mm}$ and $b=79.8 \mathrm{~mm}$, respectively. The foci of this ellipse are at $( \pm 115,0,0)$ and the reflector is truncated at $100 \mathrm{~mm}$ from $\mathrm{F} 1$, or $(-10,0,0)$.

In the laboratory, this reflector is immersed in a bath of water and objects can be placed at the second focus of the ellipsoid, F2. This device is in use at the Center for Industrial and Medical Ultrasound (CIMU) at the University of Washington Applied Physics Laboratory and we have used this geometry in order to compare directly with some laboratory experiments. Some preliminary comparisons were presented in [19].

Computationally, we use this geometry to calculate the initial condition by solving two-dimensional axisymmetric Euler equations with the Tammann equation of state (see Section 2). These initial conditions are then fed into a full three-dimensional calculation near the focus at F2.

In addition to the HM3, we have also used the geometry of the hand-held Sanywave device used in clinical studies by our collaborator Dr. Michael Chang. Some sample calculations related to the study of HOs are presented in Section 5.4.

In each case, the ESWT pressure wave form that is generated has a similar shape. There is a sharp increase in pressure from atmospheric pressure $(\sim 0.1 \mathrm{MPa})$ to a peak pressure ranging from 35 to $100 \mathrm{MPa}$ over a very short rise time $(\sim 10 \mathrm{~ns})$, followed by a decrease in pressure to $\sim-10 \mathrm{MPa}$ over $\sim 5 \mu \mathrm{s}$. The negative fluid pressure in the tail can lead to cavitation bubbles, as discussed below.

Bone healing is thought to be regulated in part by mechanical factors $[39 ; 53 ; 45$; $26 ; 23 ; 53]$. Several studies have shown that the application of cyclic compressive and shear displacements can enhance healing through increased callus formation and ossification $[39 ; 45 ; 46 ; 50 ; 43 ; 56]$. The results also indicate that treatment is also dependent upon the rate, mode and magnitude of the stress deposition [39], as well as the gap size [14].

Carter et al. [11], as well as Claes and Heigele [13], proposed a model for skeletal tissue development based on hydrostatic pressure and tensile displacements [13]. Other research has proposed a different model for skeletal tissue formation based on shear strain and fluid flow [44;30]. Augat et al. [2] found that tensile displacements are not effective in enhancing bone formation. This was further validated when Isaksson et al. [27] investigated the models in $[13 ; 11 ; 44 ; 30]$ and found that shear strain and fluid flow, were more accurate predictors of bone growth. However, no single model was able to predict certain features of the bone formation and healing process [39], highlighting the need for further research in this area. 
The shear waves generated at the fluid/solid interface have also been shown to be important in the effective break up of kidney stones $[49 ; 20]$. An additional effect of ESWT is the formation and collapse of cavitation bubbles that can cause tissue damage. While the shock wave is a compression wave, it is followed by a rarefaction wave of expansion, and in the tail the fluid pressure typically drops to negative values. Reflection at interfaces can lead to enhanced regions of expansion and to sufficiently negative pressures that cavitation bubbles can form $[38 ; 51 ; 17]$.

To better understand all of these effects, it is desirable to have a three-dimensional computational model that can simulate the focusing of nonlinear shock waves and their interaction with arbitrarily complex interfaces between different materials.

In this paper we present an approach to this problem that has allowed the study of some of these issues in a simplified context. In particular, we consider an idealized situation in which soft tissue is replaced by water, ignoring its viscoelastic properties, and modeled by the nonlinear compressible Euler equations with the Tammann or Tait equation of state. This has been used for prior ESWT work in water as well as biological-like materials [28; 41]. Bone is modeled as an isotropic and homogeneous linear elastic material [21; 29].

In reality, soft tissue and bone are very complex multiscale materials with microstructures, inhomogeneities, and anisotropic properties. Any attempt to model the biological effect of shock wave propagation through such materials may require a more sophisticated and detailed model than used here. However, we believe that many of the macroscale shock propagation issues discussed above can be adequately and most efficiently studied with a simplified model of the form considered here, since the dominant effect we hope to capture is the reflection and transmission of waves at interfaces between materials.

The compressible Euler equations with the Tammann equation of state (see Section 2.1) in two-dimensional axisymmetric geometry is used to model the initial formation of the focusing shock wave. These initial conditions are then fed into a code that uses a simpler nonlinear model, the Tait equation of state, in a threedimensional simulation of the fluid. The compressible fluid equations are written using a Lagrangian formulation that easily couples to the isotropic linear elasticity equations used in the bone-like material. The resulting equations have the same form everywhere, with a different stress-strain relationship in the different materials.

A high-resolution finite volume method is used to solve these equations. We use the wave-propagation algorithms described in [35] and implemented in Clawpack [15]. These are Godunov-type methods for the hyperbolic system that use solutions to the Riemann problem between adjacent grid cells to determine a set of waves used to update the solution, and second-order correction terms with slope limiters are added to resolve the nearly discontinuous shock waves with minimal smearing or nonphysical oscillation. 
These methods are used on a purely rectangular Cartesian grid. Each grid cell has associated with it a set of material parameters determining the material in the cell, in a unified manner so that both fluid and solid can be modeled. Complex geometry is handled by using appropriate averaged values of these parameters in cells that are cut by the interface. This is described further in Section 4.4. Averaging across the interface works quite well when the material properties are sufficiently similar and in Section 4.4 we show that this is the case even for fluid/solid boundaries of the type we consider.

We also use patch-based adaptive mesh refinement (AMR) to concentrate grid cells in regions where they are most needed to resolve features of interest. The Clawpack software contains AMR software in both two and three space dimensions and this software has been used directly for the two-dimensional axisymmetric computations of the initial shock wave described in Section 5. For the three-dimensional problem we have used ChomboClaw [10], an interface between Clawpack and the Chombo code [1] developed at the Lawrence Berkeley National Laboratory (LBL), which provides an implementation of AMR on parallel machines using MPI. Using ChomboClaw, the code originally developed using Clawpack was easily converted into a code that was run on an NSF TeraGrid machine at Texas Advanced Computing Center (TACC) and tested using up to 128 processors.

Extensive laboratory experiments have been performed on shock wave devices to measure the wave form of shock waves produced by various devices, the shape of the focal region, the peak amplitudes of pressure observed in these regions, and other related quantities. Most of these experiments have been done in a water tank where the shock wave propagates and focuses in a homogeneous medium where measurements are easily done, or with phantoms (acrylic objects with well understood photoelastic properties) that are placed in the water as a proxy for bones or kidney stones, with instrumentation such as pressure gauges or photographs used to explore the interaction of the shock wave with the object. In some cases high-speed photographs of the shock wave have been obtained. Creating phantoms from clear birefringent materials and using polarized light it is even possible to photograph the shock wave propagating through the object [48]. We have used some of these experiments to help validate our numerical approach [19].

Other researchers have also developed computational models for shock wave therapy and lithotripsy. In prior work the pressure field has been modeled using linear and nonlinear acoustics as well as the Euler equations with the Tait equation of state. Hamilton [24] used linear geometrical acoustics, which holds under the assumption of weak shock strength, to calculate the reflection of the spherical wave. The diffraction of the wave at the corner of the reflector was calculated using the Kirchoff integral method. Christopher's model [12] of the HM3 lithotripter used Hamilton's result as a starting point and considered nonplanar sources. Coleman 
et al. [17], Averkiou and Cleveland [3] used models based on the KZK equation. Tanguay [51] solved the full Euler equations and incorporated cavitation effects as well as the edge wave.

Our approach differs from these in that we consider the wave propagation in both the fluid and solid by solving a single set of equations that can model both materials. This approach allows us to investigate not only compression and tension effects of ESWT, but also the propagation of shear waves in the solid. Sapozhnikov and Cleveland [16] have investigated the effect of shear waves on spherical and cylindrical stones using linear elasticity with a plane wave initial condition. This initial condition is an unfocused wave, which yields good results for small objects, but would fail to capture the full ESWT pressure wave interaction with threedimensional bone geometries.

\section{Model equations}

To accurately model shock wave formation and propagation it is generally necessary to use nonlinear equations of compressible flow. In this work we use nonlinear equations for compressible liquids in the fluid domain (water or soft tissue) and linear elasticity in the solid domain (bone). The nonlinear compressible equations are written in a Lagrangian framework in terms of a reference configuration, as is done for the linear elasticity equations. This allows both sets of equations to be written in the same form. We apply finite volume methods to this form of the equations so that a single computational grid (or set of nested grids with AMR) can be used over the entire domain. Interfaces between fluid and solid are represented by choosing averaged material parameters in each grid cell, as discussed further in Section 4.4.

The system of equations we solve has the general form of a hyperbolic system of 9 equations

$$
q_{t}+f(q, x, y, z)_{x}+g(q, x, y, z)_{y}+h(q, x, y, z)_{z}=0,
$$

where the vector $q$ consists of the 6 components of the symmetric strain tensor followed by the momenta, and the fluxes in general may be spatially varying based on material properties:

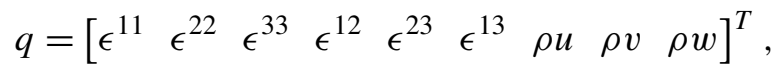

$$
\begin{aligned}
& f(q, x, y, z)=\left[\begin{array}{lllllllll}
u & 0 & 0 & v / 2 & 0 & w / 2 & \sigma^{11} & \sigma^{12} & \sigma^{13}
\end{array}\right]^{T} \text {, } \\
& g(q, x, y, z)=\left[\begin{array}{lllllllll}
0 & v & 0 & u / 2 & w / 2 & 0 & \sigma^{12} & \sigma^{22} & \sigma^{23}
\end{array}\right]^{T} \text {, } \\
& h(q, x, y, z)=\left[\begin{array}{lllllllll}
0 & 0 & w & 0 & v / 2 & u / 2 & \sigma^{13} & \sigma^{23} & \sigma^{33}
\end{array}\right]^{T} \text {. }
\end{aligned}
$$

In these expressions, ${ }^{T}$ denotes transposition, $\rho=\rho(x, y, z)$ is the density of the 
material (the "background density" independent of the wave propagating through the material) and the stress tensor $\sigma=\sigma(q, x, y, z)$ is in general a spatially varying function of $q$, linear in the solid and nonlinear in the fluid.

Within the fluid domain $\sigma=-p I$, where $p$ is the scalar pressure and $I$ is the identity matrix. The pressure is a nonlinear function of the strain as discussed further below. In the solid domain, $\sigma$ is a linear function of $\epsilon$ and is nondiagonal, allowing us to model the propagation of shear waves as well as compressional waves.

In Section 2.1 below we present the compressible fluid equations in their standard Eulerian form (the Euler equations) and discuss two possible equations of state, the Tammann EOS and the simpler Tait EOS in which the pressure is a function of density (or strain) alone, allowing us to drop the energy equation from the Euler equations. Then in Section 2.2 we rewrite these equations in the Lagrangian form given above. This can be done when modeling ESWT because the deformations are sufficiently small that the geometric nonlinearity of the equations can be ignored, adopting a Lagrangian frame and only considering the nonlinearity of the stressstrain relation as given by the equation of state.

In Section 2.3 we discuss the linear elasticity model used to model bone.

2.1. Compressible fluids in Eulerian form. Much of the previous work on ESWT has been centered around the use of the Euler equations with the Tait or Tammann equations of state. These equations of state are typically used for modeling underwater explosions like the spark plug source of the lithotripter device [24; 28]. In this section we discuss the full Euler equations and proceed to show why the Tait equation of state is sufficient for modeling ESWT. Since this equation of state is a function only of the density, and can be rewritten as a function of strain, we show in Section 2.2 how it can be modeled within the framework of elasticity, which enables us to model both the fluid and solid with the single system of equations given above.

In three space dimensions the Euler equations take the form

$$
\frac{\partial}{\partial t}\left[\begin{array}{c}
\rho \\
\rho u \\
\rho v \\
\rho w \\
E
\end{array}\right]+\frac{\partial}{\partial x}\left[\begin{array}{c}
\rho u \\
\rho u^{2}+p \\
\rho u v \\
\rho u w \\
u(E+p)
\end{array}\right]+\frac{\partial}{\partial y}\left[\begin{array}{c}
\rho v \\
\rho u v \\
\rho u^{2}+p \\
\rho v w \\
v(E+p)
\end{array}\right]+\frac{\partial}{\partial z}\left[\begin{array}{c}
\rho v \\
\rho u w \\
\rho v w \\
\rho w^{2}+p \\
w(E+p)
\end{array}\right]=0 .
$$

The total energy is $E=\rho e+\frac{1}{2}\left(u^{2}+v^{2}+w^{2}\right)$.

Several of the problems we investigated are axially symmetric and this enabled us to reduce the three-dimensional equations to a two-dimensional form. If we first rewrite the equations in cylindrical coordinates $(r, \theta, z)$ and assume no variation and 
zero velocity in the $\theta$ direction, the system we obtain is reduced to two variables, $r$ and $z$. The equations are

$$
\frac{\partial}{\partial t}\left[\begin{array}{c}
\rho \\
\rho u_{r} \\
\rho w_{z} \\
E
\end{array}\right]+\frac{\partial}{\partial r}\left[\begin{array}{c}
\rho u_{r} \\
\rho u_{r}^{2}+p \\
\rho u_{r} w_{z} \\
u_{r}(E+p)
\end{array}\right]+\frac{\partial}{\partial z}\left[\begin{array}{c}
\rho w_{z} \\
\rho u_{r} w_{z} \\
\rho w_{z}^{2}+p \\
w_{z}(E+p)
\end{array}\right]=\left[\begin{array}{c}
-\left(\rho u_{r}\right) / r \\
-\left(\rho u_{r}^{2}\right) / r \\
-\left(\rho u_{r} w_{z}\right) \\
u_{r}(E+p) / r
\end{array}\right],
$$

where $u_{r}$ and $w_{z}$ denote the velocities in the $r$ and $z$ directions. These equations are of the same form as the two-dimensional Euler equations, with the addition of geometric source terms that are a result of the variable transformation. The source terms are never evaluate at $r=0$ since we are using a finite volume method where quantities are evaluated at cell-centers, that is, the smallest value of $r$ in a calculation is $\Delta x / 2$. We prefer to keep the equations in conservation form, so they can be efficiently solved using finite volume methods.

In order to solve the system (3) or (4), we need to close the system with a relation between the pressure and conserved variables. The Tammann EOS [28] is applicable to a wide range of liquids, even with very strong shock waves. This equation of state has the form

$$
p=p(\rho, e)=(\gamma-1) \rho e-\gamma p_{\infty},
$$

where $p, \rho$ and $e$ are the pressure, density and specific internal energy, respectively, while $\gamma$ and $p_{\infty}$ are constants depending on the fluid. If $p_{\infty}=0$ this is the standard EOS for an ideal gas, with $\gamma$ generally satisfying $1<\gamma<5 / 3$, while for water $\gamma \approx 7.15$ and $p_{\infty} \approx 300 \mathrm{MPa}$. For sufficiently weak shocks, this can be approximated by the Tait equation of state,

$$
p=p(\rho)=B\left[\left(\frac{\rho}{\rho_{0}}\right)^{n}-1\right],
$$

where $B$ is a pressure term that is a weak function of entropy, but is typically treated as a constant, and corresponds to $p_{\infty}$ from (5) while $n$ corresponds to $\gamma$. Here $\rho_{0}$ is the background density measured at one atmospheric pressure. In our work we take $B=300 \mathrm{MPa}$ and $n=7.15$.

It has been common practice to use the Tait EOS in shock wave therapy and lithotripsy models [47; 41]. This has been justified by noting studies that show that entropy changes across the shock are very small even up to pressure jumps of $200 \mathrm{MPa}$ [41], which is beyond the range used in ESWT. To verify this assumption, we performed computational experiments to compare the Tammann and Tait equations of state for typical ESWT shock waves. Since we have used the f-wave approach in our computational model, we can solve (4) with a spatially 

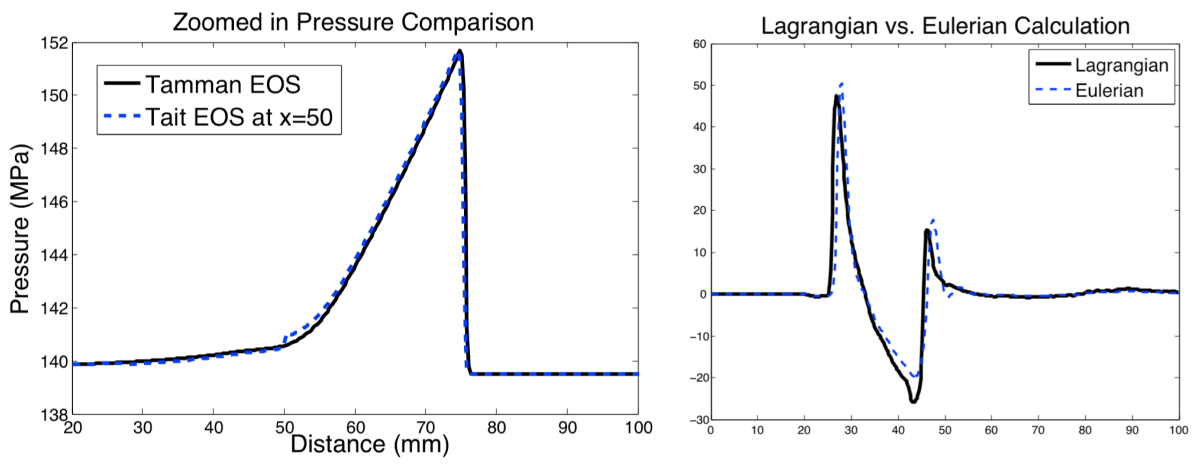

Figure 2. Left: comparison of a pressure wave calculation performed using both the Tait (blue dashed curve) and Tammann (black curve) equations of state. The results are nearly identical. Right: Comparison of the pressure pulse at F2 obtained in the Euler calculation (blue dashed curve) and the Lagrangian calculation (black curve). It is clear that the two sets of equations give good agreement. The wave in the Lagrangian case is slightly attenuated, but this may be due to error in initializing the calculation. In these calculations $\Delta x=0.5 \mathrm{~mm}$.

varying equation of state. We set up an experiment where the resulting shockwave (generated using the Tammann equation of state), was over $150 \mathrm{MPa}$. Figure 2, left, shows the results from this experiment. The black solid curve is the result from solving with the Tammann EOS in the entire domain. The blue dashed curve shows the result gotten by switching to the Tait EOS at $x=50$. This enabled us to compare the two equations of state with the exact same initial condition. There is a small disagreement at $x=50$ caused by a slight reflection at the interface due to the change in the equation of state. Otherwise, the pressure profiles are nearly identical, giving confidence that the calculations we are interested in can be done by solving the Euler equations with the Tait equation of state. This allows us to drop the equation for energy and obtain the simplified system

$$
\frac{\partial}{\partial t}\left[\begin{array}{c}
\rho \\
\rho u \\
\rho v \\
\rho w
\end{array}\right]+\frac{\partial}{\partial x}\left[\begin{array}{c}
\rho u \\
\rho u^{2}+p \\
\rho u v \\
\rho u w
\end{array}\right]+\frac{\partial}{\partial y}\left[\begin{array}{c}
\rho v \\
\rho u v \\
\rho v^{2}+p \\
\rho v w
\end{array}\right]+\frac{\partial}{\partial z}\left[\begin{array}{c}
\rho w \\
\rho u w \\
\rho v w \\
\rho w^{2}+p
\end{array}\right]=\left[\begin{array}{l}
0 \\
0 \\
0 \\
0
\end{array}\right] .
$$

2.2. Compressible fluids in Lagrangian form. In the case of a fluid where the shear modulus is zero, the stress tensor can be written as $\sigma(\epsilon)=-p I$, where $p$ is the pressure in the fluid and $I$ is the identity matrix. In the case of ESWT, the pressure only depends on changes in the density, and we can write $p(\epsilon)$ as a function of the strain tensor $\epsilon$. Consider the movement of a material with respect to a reference configuration and let $\delta=\left(\delta^{x}, \delta^{y}, \delta^{z}\right)$ be the infinitesimal displacement. 
In three space dimensions, the full strain tensor is

$$
\epsilon=\left(\begin{array}{ccc}
\delta_{x}^{x} & \frac{1}{2}\left(\delta_{y}^{x}+\delta_{x}^{y}\right) & \frac{1}{2}\left(\delta_{z}^{x}+\delta_{x}^{z}\right) \\
\frac{1}{2}\left(\delta_{y}^{x}+\delta_{x}^{y}\right) & \delta_{y}^{y} & \frac{1}{2}\left(\delta_{z}^{y}+\delta_{y}^{z}\right) \\
\frac{1}{2}\left(\delta_{x}^{z}+\delta_{z}^{x}\right) & \frac{1}{2}\left(\delta_{y}^{z}+\delta_{z}^{y}\right) & \delta_{z}^{z}
\end{array}\right),
$$

where subscripts denote partial derivatives.

In the case of small deformations, we have from conservation of mass that

$$
\rho=\frac{\rho_{0}}{1+\operatorname{tr}(\epsilon)}
$$

where $\rho_{0}$ is the equilibrium density.

If we insert this into the Tait equation of state (6) we get

$$
p(\epsilon)=B\left[\left(\frac{1}{1+\operatorname{tr}(\epsilon)}\right)^{n}-1\right] .
$$

Using the Lagrangian form is only valid in the case where the displacements are small, so we calculated the maximum value of the displacements in a twodimensional axisymmetric calculation with the Euler equations. We found that for a maximum peak pressure of $50 \mathrm{MPa}$, the corresponding maximum velocity was $10^{-3} \mathrm{~m} / \mathrm{s}$. We then calculated the maximum displacement by integrating the velocity over the time of the calculation and found this to be on the order of $10^{-5} \mathrm{~mm}$. The size of the grid cell is on the order of $10^{-1} \mathrm{~mm}$, so the displacements are 4 orders of magnitude smaller than the width of the grid cells. It is therefore reasonable to assume that the density in each grid cell is essentially constant and that the Lagrangian framework of the elasticity equations will be valid for the fluid.

To test this, we took the same initial condition for the two-dimensional axisymmetric Euler equations with the Tammann equation of state and the corresponding two-dimensional axisymmetric Lagrangian form of the equations with the Tait equation of state and measured the pressure at the focus, F2. The results in Figure 2, right, demonstrate reasonably good agreement between the two cases, but the Lagrangian form is slightly attenuated. This may be due to conversion of the initial condition from the conserved variables in the Euler equations (4) to those in the elasticity equations (1).

Since the displacements are small, we also considered the possibility that nonlinearity in the fluid could be ignored, so we could instead use a linearized version of the Tait equation of state. Then we would be able to simply use the linear elasticity equations throughout the domain, in both the fluid and solid materials. If we assume a small perturbation to the strain, $\epsilon+\delta \epsilon$, we can expand the Tait EOS (6) as a Taylor series about $\epsilon$, 


$$
p(\epsilon+\delta \epsilon)=p_{0}+p^{\prime}(\delta \epsilon) \epsilon+\frac{p^{\prime \prime}(\delta \epsilon)}{2} \epsilon^{2}+\cdots .
$$

If we keep the first two terms of the expansion, the EOS has been linearized and we will call this the linear Tait EOS. Similarly, we will refer to the equation obtained by keeping the first three terms of the expansion as the quadratic Tait EOS. Onedimensional tests of both possibilities are shown in Figure 3, for three different wave amplitudes. For a wave with maximum amplitude less than $3 \mathrm{MPa}$ there is fairly
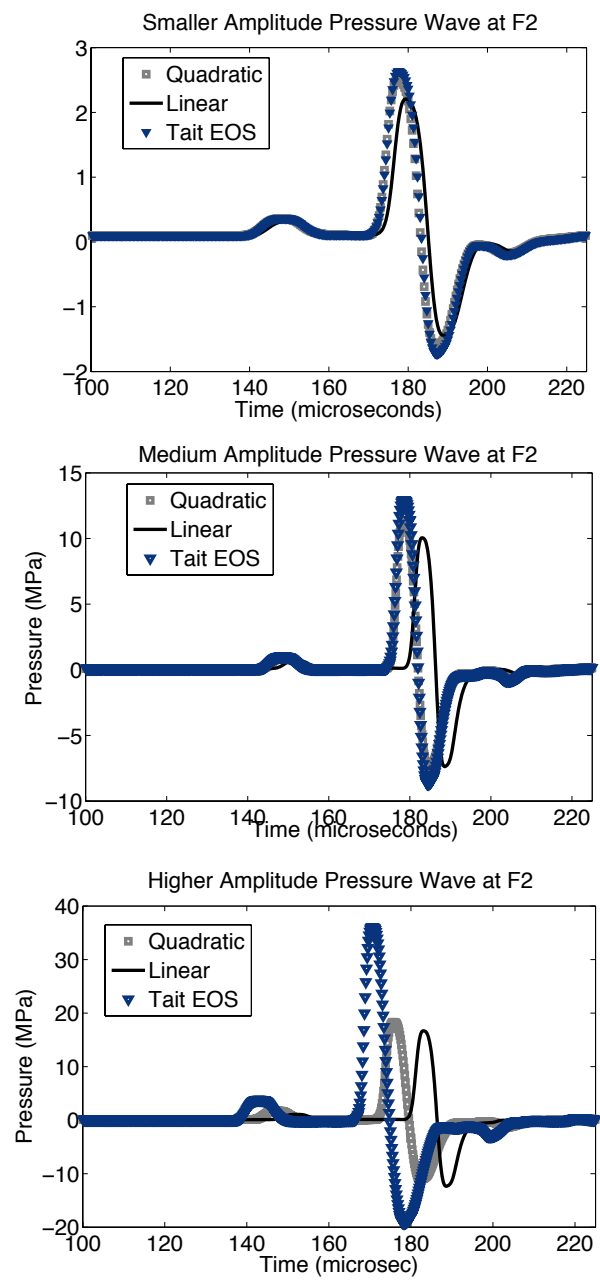

Figure 3. Pressure gauge measurement at F2 of different versions of the Tait EOS at different amplitudes. The triangular markers indicate the full nonlinear Tait EOS, the solid line is a linearized version and the square markers are a quadratic version. The linearized versions of the EOS work reasonably well at small amplitudes, but it's clear from the bottom figure that as the pressures increase to those observed in ESWT, the full nonlinear equation of state must be used. 
good agreement, however, as the amplitude is increased, as is required for ESWT, the linear and quadratic equations of state do not capture the correct behavior. Thus we used the full Tait EOS in the fluid domain.

2.3. Elasticity equations. In the current work we model bone as a linear isotropic solid. We use the equations (1) together with Hooke's law

$$
\begin{aligned}
\sigma^{11} & =C_{11} \epsilon^{11}+C_{12} \epsilon^{22}+C_{13} \epsilon^{33}, \\
\sigma^{22} & =C_{21} \epsilon^{11}+C_{22} \epsilon^{22}+C_{23} \epsilon^{33}, \\
\sigma^{33} & =C_{31} \epsilon^{11}+C_{32} \epsilon^{22}+C_{33} \epsilon^{33}, \\
\sigma^{12} & =C_{44} \epsilon^{12}, \\
\sigma^{13} & =C_{55} \epsilon^{13}, \\
\sigma^{23} & =C_{66} \epsilon^{23},
\end{aligned}
$$

where the spatially varying scalar coefficients $C_{i j}(x, y, z)$ are determined by the properties of the material being modeled. The parameters used for the bone model were found in [37].

For an isotropic material we can relate the $C_{i j}$ above to the two Lamé parameters, $\lambda$ and $\mu$, that are used to model different elastic materials. $C_{i i}=\lambda+2 \mu$ for $i=1, \ldots, 3, C_{i i}=2 \mu$ for $i=4, \ldots, 6$, and $C_{i j}=\lambda$ for $i \neq j$. Here $\mu$ is the shear modulus and $\lambda+2 \mu$ is the bulk modulus of the material. Note that the $\lambda$ here is different from the $\lambda^{i}$ used to denote the eigenvalues elsewhere in the paper.

Linear elasticity has been used extensively in the literature to model both trabecular and cortical bone [29;21]. Linear viscoelastic models have also been used for ultrasound wave propagation in bone [22]. Our model could be extended to orthotropic models, requiring 9 material parameters, as has also been used for bone modeling; see, for example, [52].

\section{Eigenstructure of the hyperbolic system}

The full three-dimensional system of equations (1) models both the nonlinear fluid and the linear elastic bone as described in the preceding sections. This system can be written in quasilinear form:

$$
q_{t}+A(q, x, y, z) q_{x}+B(q, x, y, z) q_{y}+C(q, x, y, z) q_{z}=0,
$$

where $A, B$ and $C$ are the Jacobians of the flux functions in the $x, y$ and $z$ directions respectively. For the multidimensional methods implemented in Clawpack, we need the solution to the Riemann problem along slices in each coordinate direction. Here we provide the details for the solution in the $x$ direction, but the solution in the $y$ and $z$ directions are similar with appropriate permutations to the $B$ and $C$ matrices. 
The corresponding Jacobian for this system in the $x$ direction is:

$$
\begin{aligned}
& A(q, x, y, z)=\frac{\partial f(q, x, y, z)}{\partial x} \\
& =-\left(\begin{array}{ccccccccc}
0 & 0 & 0 & 0 & 0 & 0 & \frac{1}{\rho_{0}} & 0 & 0 \\
0 & 0 & 0 & 0 & 0 & 0 & 0 & 0 & 0 \\
0 & 0 & 0 & 0 & 0 & 0 & 0 & 0 & 0 \\
0 & 0 & 0 & 0 & 0 & 0 & 0 & \frac{1}{2 \rho_{0}} & 0 \\
0 & 0 & 0 & 0 & 0 & 0 & 0 & 0 & 0 \\
0 & 0 & 0 & 0 & 0 & 0 & 0 & 0 & \frac{1}{2 \rho_{0}} \\
\sigma_{\epsilon^{11}}^{11} & \sigma_{\epsilon^{22}}^{11} & \sigma_{\epsilon^{33}}^{11} & 0 & 0 & 0 & 0 & 0 & 0 \\
0 & 0 & 0 & \sigma_{\epsilon^{12}}^{12} & 0 & 0 & 0 & 0 & 0 \\
0 & 0 & 0 & 0 & 0 & \sigma_{\epsilon^{13}}^{13} & 0 & 0 & 0
\end{array}\right),
\end{aligned}
$$

where $\sigma_{\epsilon^{33}}^{11}$, for example, denotes the partial derivative of $\sigma^{11}$ with respect to $\epsilon^{33}$. In the linear elastic case this is simply the coefficient $C_{13}$, but the above form also applies to the nonlinear compressible equations. The spatial variation in $f(q, x, y, z)$ and the Jacobian $A$ result from allowing the material parameters such as density and elastic moduli to vary in space. The Jacobians in the $y$ and $z$ directions are similar with the entries permuted appropriately.

The eigenvalues for system (19) are

$$
\lambda^{1,2}= \pm \sqrt{\frac{\sigma_{\epsilon^{11}}^{11}}{\rho_{0}}} ; \quad \lambda^{3,4}= \pm \sqrt{\frac{\sigma_{\epsilon^{12}}^{12}}{2 \rho_{0}}} ; \quad \lambda^{5,6}= \pm \sqrt{\frac{\sigma_{\epsilon^{13}}^{13}}{2 \rho_{0}}} ; \quad \lambda^{7,8,9}=0 .
$$

When modeling a fluid where the shear stress is zero, there are seven zero-speed eigenvalues since $\sigma_{\epsilon^{12}}^{12}=\sigma_{\epsilon^{13}}^{13}=0$. Only the compressional waves corresponding to $\lambda^{1,2}$ propagate with nonzero speed. Note that the Tait equation of state (10) gives

$$
\sigma_{\epsilon^{11}}^{11}=\frac{\partial \sigma^{11}}{\partial \epsilon^{11}}=B n\left(\frac{1}{1+\epsilon^{11}+\epsilon^{22}+\epsilon^{33}}\right)^{n+1}=\frac{n(p+B)}{1+\operatorname{tr} \epsilon} .
$$

In the small amplitude acoustic limit $\epsilon \rightarrow 0$, from (20) we obtain the wave speeds

$$
\pm \sqrt{\frac{n(p+B)}{\rho_{0}}},
$$

which are the expected waves speeds for compressional waves in the Lagrangian form with this equation of state.

For the elastic solid, on the other hand, waves 1 and 2 correspond to P-waves while waves 4-6 correspond to $S$-waves, and the expected wave speeds are recovered 
based on the elastic coefficients given in Section 2.3. For example, in the $x$ direction the P-wave speeds are

$$
\pm \sqrt{\frac{C_{11}}{\rho_{0}}}
$$

and the S-wave speeds are

$$
\pm \sqrt{\frac{C_{44}}{2 \rho_{0}}} \text { and } \pm \sqrt{\frac{C_{55}}{2 \rho_{0}}} .
$$

The corresponding eigenvectors for system (19) are

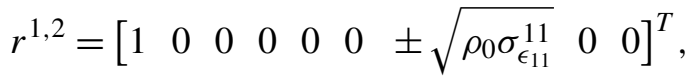

$$
\begin{aligned}
& r^{3,4}=\left[\begin{array}{lllllllll}
0 & 0 & 0 & 1 & 0 & 0 & 0 & \pm \sqrt{2 \rho_{0} \sigma_{\epsilon^{12}}^{12}} & 0
\end{array}\right]^{T}, \\
& r^{5,6}=\left[\begin{array}{lllllllll}
0 & 0 & 0 & 0 & 0 & 1 & 0 & 0 & \pm \sqrt{2 \rho_{0} \sigma_{\epsilon_{13}}^{13}}
\end{array}\right]^{T},
\end{aligned}
$$

for the P-waves and S-waves, and

$$
\begin{aligned}
r^{7} & =\left[\begin{array}{lllllllll}
-\sigma_{\epsilon^{22}}^{11} & \sigma_{\epsilon^{11}}^{11} & 0 & 0 & 0 & 0 & 0 & 0 & 0
\end{array}\right]^{T}, \\
r^{8} & =\left[\begin{array}{lllllllll}
-\sigma_{\epsilon^{33}}^{11} & 0 & \sigma_{\epsilon^{11}}^{11} & 0 & 0 & 0 & 0 & 0 & 0
\end{array}\right]^{T}, \\
r^{9} & =\left[\begin{array}{lllllllll}
0 & 0 & 0 & 0 & 1 & 0 & 0 & 0 & 0
\end{array}\right]^{T},
\end{aligned}
$$

for the stationary waves.

3.1. Axisymmetric form of the equations. We used the two-dimensional axisymmetric form of the equations to generate an initial condition for our three-dimensional calculations, as well as for validation of our model.

The three-dimensional equations in cylindrical coordinates are:

$$
\begin{gathered}
\epsilon_{t}^{r r}=\frac{\partial u}{\partial r}, \quad \epsilon_{t}^{\theta \theta}=\frac{u}{r}+\frac{1}{r} \frac{\partial v}{\partial \theta}, \quad \epsilon_{t}^{z z}=\frac{\partial w}{\partial z} \\
\epsilon_{t}^{r z}=\frac{1}{2}\left(\frac{\partial u}{\partial z}+\frac{\partial w}{\partial r}\right), \quad \epsilon_{t}^{r \theta}=\frac{1}{2}\left(\frac{\partial v}{\partial r}+\frac{1}{r} \frac{\partial u}{\partial \theta}-\frac{v}{r}\right), \quad \epsilon_{t}^{\theta z}=\frac{1}{2 r}\left(\frac{\partial w}{\partial \theta}+\frac{\partial v}{\partial z}\right), \\
\rho u_{t}=\frac{1}{r} \frac{\partial \sigma^{r \theta}}{\partial \theta}+\frac{\partial \sigma^{r r}}{\partial r}+\frac{\sigma^{r r}-\sigma^{\theta \theta}}{r}+\frac{\partial \sigma^{r z}}{\partial z}, \\
\rho v_{t}=\frac{1}{r} \frac{\partial \sigma^{\theta \theta}}{\partial \theta}+\frac{\partial \sigma^{r \theta}}{\partial r}+\frac{2 \sigma^{r \theta}}{r}+\frac{\partial \sigma^{z \theta}}{\partial z}, \\
\rho w_{t}=\frac{1}{r} \frac{\partial \sigma^{z \theta}}{\partial \theta}+\frac{\partial \sigma^{z z}}{\partial z}+\frac{\partial \sigma^{r z}}{\partial r}+\frac{\sigma^{r z}}{r} .
\end{gathered}
$$


If we assume that $v=\epsilon_{\theta z}=\epsilon_{r \theta}=0$ and there is no variation in the $\theta$ direction, then the system (27) simplifies to

$$
\begin{aligned}
\epsilon_{t}^{r r}=\frac{\partial u}{\partial r}, \quad \epsilon_{t}^{\theta \theta} & =\frac{u}{r}, \quad \epsilon_{t}^{z z}=\frac{\partial w}{\partial z}, \quad \epsilon_{t}^{r z}=\frac{1}{2}\left(\frac{\partial u}{\partial z}+\frac{\partial w}{\partial r}\right), \\
\rho u_{t} & =\frac{\partial \sigma^{r r}}{\partial r}+\frac{\sigma^{r r}-\sigma^{\theta \theta}}{r}+\frac{\partial \sigma^{r z}}{\partial z}, \\
\rho w_{t} & =\frac{\partial \sigma^{z z}}{\partial z}+\frac{\partial \sigma^{r z}}{\partial r}+\frac{\sigma^{r z}}{r} .
\end{aligned}
$$

It is interesting to note here that the strain in the $\theta \theta$ direction is nonzero and in this case is called the hoop strain. A uniform radial displacement is not a rigid body motion, as it would be in the two-dimensional plane strain case, but instead produces a circumferential strain. This is because the original circumference of the cylinder is $2 \pi r$, but when there is a strain in the radial direction the circumference grows to $2 \pi\left(r+u_{r}\right)$, inducing a strain $2 \pi u_{r} / 2 \pi r=u_{r} / r$.

The Jacobian for system (28) in the $z$ direction is

$$
f^{\prime}(q)=-\left(\begin{array}{cccccc}
0 & 0 & 0 & 0 & \frac{1}{\rho_{0}} & 0 \\
0 & 0 & 0 & 0 & 0 & 0 \\
0 & 0 & 0 & 0 & 0 & 0 \\
0 & 0 & 0 & 0 & 0 & \frac{1}{2 \rho_{0}} \\
\sigma_{\epsilon_{r r}}^{r r} & \sigma_{\epsilon_{z z}}^{r r} & 0 & 0 & 0 & 0 \\
0 & 0 & 0 & \sigma_{\epsilon_{r z}}^{r z} & 0 & 0
\end{array}\right),
$$

and has an eigenstructure that is equivalent to the two-dimensional elasticity equations, with the addition of a second zero-speed eigenvalue.

These equations have the structure

$$
q_{t}+f(q)_{r}+g(q)_{z}=S(q, r)
$$

with source terms

$$
\epsilon_{t}^{\theta \theta}=\frac{u}{r}, \quad \rho u_{t}=\frac{\sigma_{r r}-\sigma_{\theta \theta}}{r}, \quad \rho w_{t}=\frac{\sigma_{r z}}{r} .
$$

In Clawpack, we solve these equations with a fractional-step method. The full problem is split into two subproblems that are solved independently. We first solve the homogeneous system obtained by setting $S \equiv 0$ in (30) using the wave propagation algorithm described in Section 4, and then solve

$$
q_{t}=S(q, r),
$$

with an appropriate ODE solver. For (31), we use forward Euler. 


\section{Numerical methodology}

We used the wave-propagation algorithms described in [35] and implemented in Clawpack [15] to solve the hyperbolic systems of PDEs derived in the preceding sections. In this section we provide the basic details of the numerical methodology and the approximate solution to the Riemann problem with a spatially varying flux function, similar to what was done in [36]. We also discuss computational issues that require the use of adaptive mesh refinement.

4.1. Riemann solvers and wave-propagation algorithms. Recall that the "Riemann problem" is the initial value problem for a one-dimensional hyperbolic system of the form

$$
q_{t}+f(q, x)_{x}=0
$$

with special initial data consisting of two constant states separated by a discontinuity

$$
q_{0}(x)= \begin{cases}Q_{l} & \text { if } x<0 \\ Q_{r} & \text { if } x>0\end{cases}
$$

If the flux function is spatially varying then we also use a piecewise-defined flux function with

$$
f(q, x)= \begin{cases}f_{l}(q) & \text { if } x<0 \\ f_{r}(q) & \text { if } x>0\end{cases}
$$

The Riemann problem plays a fundamental role in the theory and computation of hyperbolic problems, since the Riemann solution consists of waves propagating at constant speeds and can generally be computed. For nonlinear systems of equations this is often replaced by an approximate Riemann solver as will be discussed below.

For a linear system of equations $q_{t}+A(x) q_{x}=0$ the Riemann solution is easily computed in terms of the eigenvectors and eigenvalues of the matrices $A_{l}$ to the left of the interface and $A_{r}$ to the right of the interface. We begin by discussing the linear case with a constant matrix $A$ and turn to the variable-coefficient (heterogeneous media) case in Section 4.3. We assume the matrix $A$ is diagonalizable,

$$
A=R \Lambda R^{-1}
$$

where $R$ is the matrix of eigenvectors and $\Lambda$ is the diagonal matrix of eigenvalues. The Riemann solution is computed by decomposing $\Delta Q=Q_{r}-Q_{l}$ as a linear combination of eigenvectors of $A$,

$$
\Delta Q=\sum_{p=1}^{m} \alpha^{p} r^{p}, \quad \text { where } \alpha=R^{-1} \Delta Q .
$$

We denote the $p$-th wave by $\mathcal{W}_{p}=\alpha^{p} r^{p}$, where $p=1,2, \ldots, m$ and the number of waves $m$ is equal to the number of equations in the system. 
We use finite volume methods in which $Q_{i}^{n}$ represents a cell average of the vector $q$ in cell $i$ at time $t_{n}$ (still in one space dimension). In Godunov's method the cell average is updated by the waves entering the cell from the interfaces to the left and the right, and each wave updates the cell average by $\mathscr{W}^{p}$, the jump in $q$ across the wave, multiplied by the distance the wave propagates over the time step and divided by the length of the cell, that is, the cell average is updated by $\left(\lambda^{p} \Delta t / \Delta x\right)^{q} W^{p}$. To express the total update to a cell, it is convenient to define matrices $A^{+}$and $A^{-}$via

$$
A^{ \pm}=R \Lambda^{ \pm} R^{-1}, \quad \text { where } \Lambda^{ \pm}=\operatorname{diag}\left(\lambda_{p}^{ \pm}\right),
$$

with $\lambda^{+}=\max (\lambda, 0)$ and $\lambda^{-}=\min (\lambda, 0)$. Then the cell average is updated by

$$
Q_{i}^{n+1}=Q_{i}^{n}-\frac{\Delta t}{\Delta x}\left(A^{+} \Delta Q_{i-1 / 2}+A^{-} \Delta Q_{i+1 / 2}\right) .
$$

Here $\Delta Q_{i-1 / 2}=Q_{i}-Q_{i-1}$ is the jump across the interface at $i-1 / 2$, for example. For a linear system this is a generalization of the upwind method and is first order accurate.

Second order accuracy is achieved by adding in correction fluxes:

$$
Q_{i}^{n+1}=Q_{i}^{n}-\frac{\Delta t}{\Delta x}\left(A^{+} \Delta Q+A^{-} \Delta Q\right)-\frac{\Delta t}{\Delta x}\left(\tilde{F}_{i+1 / 2}-\tilde{F}_{i-1 / 2}\right),
$$

where

$$
\tilde{F}_{i-1 / 2}=\frac{1}{2}\left(1-\left|\frac{\lambda^{p} \Delta t}{\Delta x}\right|\right)\left|\lambda^{p}\right| W_{i-1 / 2}^{p} .
$$

These terms convert the upwind method into a method of Lax-Wendroff type, matching terms through $\Delta t^{2} A^{2} q_{x x}$ in the Taylor series expansion of the solution at the end of the time step. This method generates dispersive errors, however, that can create large nonphysical oscillations near steep gradients or discontinuities in a solution, such as shock waves. To turn this into a "high-resolution" method, we use a wave limiter, replacing $\mathcal{W}_{i-1 / 2}^{p}$ in (41) by $\widetilde{W}_{i-1 / 2}^{p}$, a limited version of the wave. The wave $\mathcal{W}_{i-1 / 2}^{p}$ is compared to the corresponding wave from the neighboring Riemann problem, either $W_{i-3 / 2}^{p}$ if $\lambda^{p}>0$ or $W_{i+1 / 2}^{p}$ if $\lambda^{p}<0$. If the waves are of comparable magnitude the full correction term is used for accuracy, but if there is a large discrepancy then the solution is not smooth at this point and a limited version is applied. See [34] or [35, Chapter 6] for more complete details.

In two or three space dimensions the idea is the same, but now a one-dimensional Riemann problem must be solved normal to each edge or face of the cell. The resulting waves update the cell averages and correction fluxes analogous to (41) are used along with limiters in each direction.

In addition, to achieve second-order accuracy and good stability properties, it is also necessary to use "transverse Riemann solvers" that further modify the correction fluxes $\tilde{F}$ at each cell edge. The method described above is based on 
propagating waves normal to each interface. In reality, the waves will propagate in a multidimensional manner and affect cell averages in cells above and below those that are directly adjacent to the interface.

In two dimensions, each "fluctuation" such as $A^{-} \Delta Q_{i-1 / 2, j}$ and $A^{+} \Delta Q_{i-1 / 2, j}$ that results from solving a Riemann problem in the $x$ direction is split into two pieces using the eigenstructure of the coefficient matrix $B$ in the $y$ direction, for example:

$$
A^{+} \Delta Q_{i-1 / 2, j}=B^{-} A^{+} \Delta Q_{i-1 / 2, j}+B^{+} A^{+} \Delta Q_{i-1 / 2, j} .
$$

These two pieces will modify the correction flux at the edges $(i, j-1 / 2)$ and $(i, j+1 / 2)$ respectively to capture the transverse motion of the right-going wave. Similarly, after solving a normal Riemann problem in the $y$ direction using the $B$ matrix, transverse problems are solved based on the eigenstructure of $A$. The net effect of all these corrections is to incorporate terms modeling the cross-derivative terms $B A q_{x y}$ and $A B q_{y x}$ of the Taylor series expansion in a properly upwinded manner. More details can be found in [34] or [35, Chapter 21]. The transverse correction terms are needed for accuracy, but also have the effect of improving the stability limit, allowing a Courant number near 1 to be used, relative to the maximum wave speed in any direction.

In three space dimensions there are two transverse directions for each normal Riemann solve, and terms modeling $C A q_{x z}$, etc. must also be included. Moreover, "double transverse" terms must be included, splitting the result of a transverse solve into eigenvectors of the remaining coefficient matrix, and modeling terms such as $B C A q_{x z y}$. The details are presented in [31] and fully implemented in Clawpack.

4.2. The nonlinear fluid Riemann solver. The compressible fluid equations in Lagrangian form discussed in Section 2.2 can be reduced to the quasilinear form (18) in which the Jacobian matrices depend only on $q$ (for a spatially uniform fluid). To apply the wave-propagation algorithm we need to solve the Riemann problem orthogonal to each cell interface. For nonlinear problems this is usually done using an approximate Riemann solver, for example, by replacing $f(q)_{x}$ by $\hat{A} q_{x}$, where the matrix $\hat{A}$ at each cell interface is chosen based on the data $Q_{l}$ and $Q_{r}$ to the left and right. We use the f-wave formulation of the wave-propagation algorithm [4], in which the jump in flux $f\left(Q_{r}\right)-f\left(Q_{l}\right)$ is split into eigenvectors of an approximate Jacobian matrix, rather than the jump in $Q$. This leads to an algorithm that is conservative for any choice of approximate Jacobian and also extends naturally to the case of spatially varying fluxes, as required near the fluid-solid boundary and discussed further below.

Rather than choose an approximate Jacobian $\hat{A}$ and then determining its eigenvectors and eigenvalues, we simply choose the set of eigenvectors and associated 
wave speeds based on the data and wave forms expected to result from this data. These vectors form a matrix $\hat{R}$ and we then solve $\hat{R} \beta=f\left(Q_{r}\right)-f\left(Q_{l}\right)$ for the vector of wave strengths $\beta$. The choice of vectors in $\hat{R}$ and associated wave speeds $\hat{\lambda}$ implicitly defines the Jacobian approximation $\hat{A}=\hat{R} \hat{\Lambda} \hat{R}^{-1}$, but this matrix is never needed.

The eigenvectors are taken to be the vectors displayed in (25) and (26). Recall that in the fluid case there are only two nonzero eigenvalues corresponding to the first two eigenvectors. For the eigenvector corresponding to $\lambda^{1}<0$ we use $\lambda^{1}=-\sigma_{\epsilon^{11}}^{11}$ evaluated in the left state $Q_{l}$, while the eigenvector corresponding to $\lambda^{2}>0$ is determined using $\lambda^{2}=\sigma_{\epsilon^{11}}^{11}$ evaluated in the right state $Q_{r}$. These vectors have nonzero components only in positions 1 and 7 and so the values of $\beta^{1}$ and $\beta^{2}$ can be determined by solving a $2 \times 2$ system:

$$
\left[\begin{array}{cc}
1 & 1 \\
\rho_{l} \lambda_{l}^{1} & \rho_{r} \lambda_{r}^{2}
\end{array}\right]\left[\begin{array}{l}
\beta^{1} \\
\beta^{2}
\end{array}\right]=\left[\begin{array}{l}
\Delta f^{1} \\
\Delta f^{7}
\end{array}\right]
$$

The solution is

$$
\begin{aligned}
& \beta^{1}=\frac{\rho_{r} \lambda_{r}^{2} \Delta f^{1}-\Delta f^{7}}{\rho_{r} \lambda_{r}^{2}-\rho_{l} \lambda_{l}^{1}}, \\
& \beta^{2}=\frac{\Delta f^{7}-\rho_{l} \lambda_{l}^{1} \Delta f^{1}}{\rho_{r} \lambda_{r}^{2}-\rho_{l} \lambda_{l}^{1}} .
\end{aligned}
$$

The remaining waves do not propagate and do not come into the wave-propagation algorithm.

4.3. The linear elastic Riemann solver. In the linear elastic material modeling bone, we take a similar approach and again use the f-wave formulation of the algorithm. In this case there are six waves with nonzero wave speeds given by the eigenvectors in (25). The eigenvectors are independent of $q$ in the linear case, but can be spatially varying to represent varying bone structure, so the coefficients $C_{i j}$ in (12) can vary from one grid cell to the next. Similar to the nonlinear case described above, to compute the decomposition of the flux difference into propagating waves we define the three left-going eigenvectors $r^{1,3,5}$ (with the minus sign in (25)) based on the coefficients in the left state, while the right-going eigenvectors $r^{2,4,6}$ are defined using the coefficients in the right state. Note that the flux vector $f(q)$ from (2), and hence any jump in flux, has zeros in three components which are easily seen to lead to $\beta^{7}=\beta^{8}=\beta^{9}$ when the flux difference is written as a linear combination of the eigenvectors, and the six remaining components of the flux difference uniquely define the coefficients $\beta^{1}$ through $\beta^{6}$ for the six propagating waves. The weights $\beta^{1}$ and $\beta^{2}$ are the same as is (44), and the others are 


$$
\begin{aligned}
& \beta^{3}=\frac{\rho_{r} \lambda_{r}^{4} \Delta f^{4}-\Delta f^{8}}{\rho_{r} \lambda_{r}^{4}-\rho_{l} \lambda_{l}^{3}}, \quad \beta^{4}=\frac{\Delta f^{8}-\rho_{l} \lambda_{l}^{3} \Delta f^{4}}{\rho_{r} \lambda_{r}^{4}-\rho_{l} \lambda_{l}^{4}}, \\
& \beta^{5}=\frac{\rho_{r} \lambda_{r}^{6} \Delta f^{6}-\Delta f^{9}}{\rho_{r} \lambda_{r}^{6}-\rho_{l} \lambda_{l}^{5}}, \quad \beta^{6}=\frac{\Delta f^{9}-\rho_{l} \lambda_{l}^{5} \Delta f^{6}}{\rho_{r} \lambda_{r}^{6}-\rho_{l} \lambda_{l}^{5}} .
\end{aligned}
$$

4.4. Interfaces and the Cartesian grid. In ESWT the pressure wave must propagate through a variety of materials, and in general the interfaces between different materials do not align with the grid. In our calculations we use a Cartesian grid. To handle grid cells that contain two materials, we perform a weighted average of the material properties. The stress-strain relationship in the averaged grid cells is taken to be that from linear elasticity, even if one of the materials is fluid. This approach is feasible because we use AMR to refine around the interfaces between the two materials. By using a fine enough grid, we are able to reduce the error introduced by the weighted average approximation. Figure 4, left, illustrates the interface between the fluid and the brass reflector from an axisymmetric calculation. Three grid resolutions are shown in this figure: a coarse grid on the right, a level 2 grid that is refined by a factor of 4 in each direction in the middle, and the finest grid on the left, where the grid lines are not drawn.

Figure 4, right, shows a comparison between the pressure wave measured at F2 for an AMR calculation versus a single grid calculation. The single grid calculation took 269 minutes to complete, just over 6 times as long as long as the run using AMR which finished in 44 minutes. These calculations were performed serially on a $2.8 \mathrm{GHz}$ dual core AMD Opteron machine with $32 \mathrm{~GB}$ of memory. It's clear that the two calculations yield comparable pressure waves. The biggest difference is in the direct wave arriving around $t=150$, which is not being resolved in the AMR

Adaptive Grids for ESWT Reflector

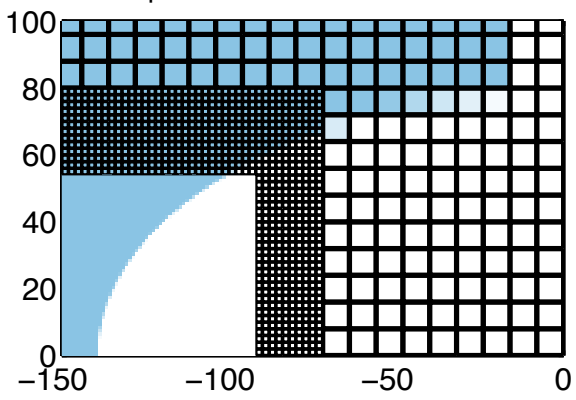

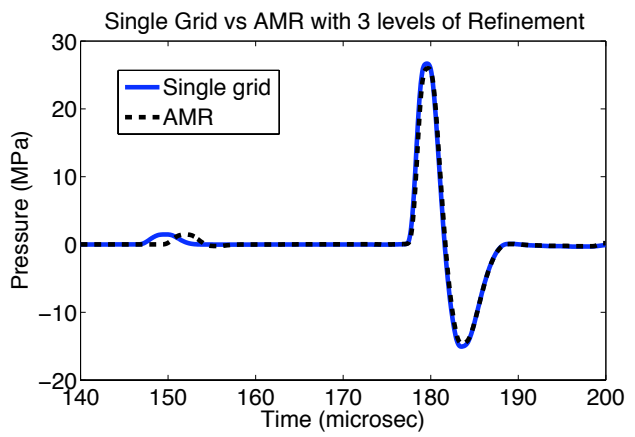

Figure 4. Left: resolution of the ellipsoid reflector with different levels of AMR. Right: two-dimensional axisymmetric calculation. The second is a comparison of the waveform obtained using AMR and a uniform grid. The finest grid resolution in the AMR calculation is the same as the resolution on the uniform grid. 
calculation because we have refined only in the vicinity of the reflected wave of primary interest.

4.5. Adaptive mesh refinement. The pressure waveform found in ESWT contains a very thin region of high pressure that can not be resolved without a highly refined mesh. In Figure 5 we investigated the effect of grid refinement on the shock wave profile and found that with grid resolution greater than $0.25 \mathrm{~mm}$, the wave form at F2 was not a shock. Note that near the shock we only expect our method to be first order, but the solution does converge to a shock as the grid is refined. Our calculations are done with the adaptive mesh refinement (AMR) in the style of Colella, Berger and Oliger [5; 8]. The AMR algorithms used in Clawpack are more fully described in [7]. For the three-dimensional calculations, a similar AMR algorithm is used, as implemented in Chombo. Here we only briefly review the main ideas.

The computational domain is covered by a rectangular level- 1 grid, typically at a coarse resolution. Rectangular patches of the grid may be covered by level-2 grids, refined by some specified refinement ratio in each direction. Since we use explicit methods, the Courant-Friedrichs-Lewy condition generally requires that the time step be refined by the same factor on the level- 2 grids, so several time steps must be taken on each level-2 grid for each time step on the level-1 grid. The level 1 grid is advanced first, and for each time step on the level-2 grid, ghost cell values around the boundary are filled in either by copying from adjacent grids at the same level, or using space-time interpolation from the level-1 grid for ghost cells that do not lie in an adjacent grid. This entire procedure is repeated recursively to obtain

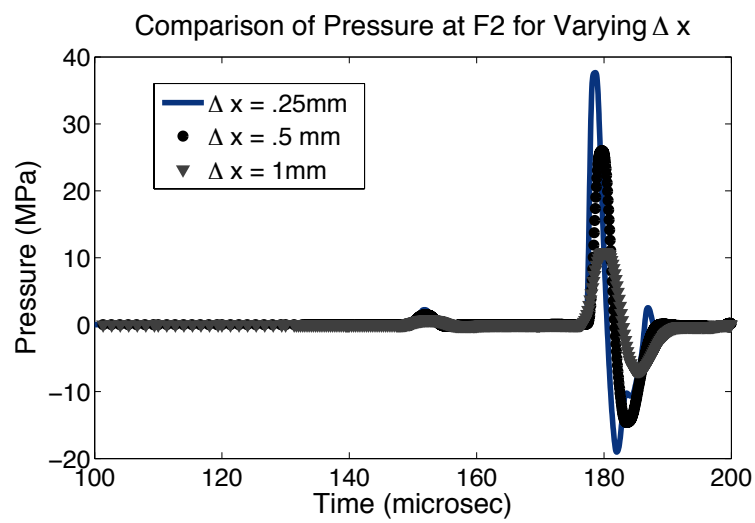

Figure 5. Effect of grid size on shock wave profile. As the grid is refined for the same initial condition, the shock wave profile steepens. The solution eventually converges to a profile with the same magnitude, though the convergence rate is only first order near a discontinuity. 
higher levels of refinement; e.g., some portion of the collection of level-2 grids may be covered by level-3 grids and so on.

In order to adaptively refine the grid, it is important to specify appropriate refinement criteria. The perturbations to the strain are small, so gradients in the strain are too small to use as reliable refinement criteria. However, the small strains result in large changes in the pressure, so we refine in the area near the pressure wave. In order to handle the interfaces between two materials, we also use large gradients in background density as a secondary refinement criterion. Cells that are flagged as needing refinement are clustered into rectangular patches using the algorithm of Berger and Rigoutsis [6]. Regridding is done every few steps on each grid level in order to track propagating waves. Regions are automatically de-refined once the wave passes by, since cells in these regions are no longer flagged as needing refinement.

Figure 5 illustrates the behavior of the ESWT waveform as the grid is refined. What is evident from these experiments is that a coarse grid will not effectively capture the development of the shock, so around the propagating wave, we need at least $\Delta x=0.25 \mathrm{~mm}$ resolution. As the wave steepens into a shock, we no longer expect second order convergence, because in the region around a discontinuity, our methods are first order. However, since the discontinuities occur in a small region of the domain, the overall methodology is still second order.

In order to efficiently calculate a reasonable ESWT waveform in three dimensions, we utilized ChomboClaw [10], which uses the adaptive mesh refinement routines of CHOMBO with the wave propagation solvers of Clawpack. This code can be run in parallel using MPI on an NSF TeraGrid computer at TACC.

\section{Results}

We have used the approach described above to model ESWT pressure waves interacting with three-dimensional bone geometries comprised of idealized materials. We have modeled both simple objects that have been used in laboratory experiments as well as complex three-dimensional geometries extracted from CT scans of patient data [18]. Here we present results that demonstrate the efficacy of the Lagrangian formulation, as well as examples of calculations performed using real three-dimensional geometries.

The calculations were initialized using pressure data obtained from a twodimensional axisymmetric calculation where we modeled the full geometry of the ellipsoidal reflector. The reflector was modeled using linear elasticity with material properties that can be found in [18]. We assumed the fluid was water with the corresponding parameters for the Tait equation of state found in Section 2.1. We saved the data at $t=116 \mu \mathrm{s}$ and used this to restart future calculations. For the 
three-dimensional initial condition, we rotated the two-dimensional data about the $x$-axis. The material properties of averaged bone were obtained from [37] and used in the heterotopic ossification, cylinder and sphere calculations.

We have found in our experiments that interfaces between materials with large impedance differences have the most significant effect on maximum stress and energy deposition.

5.1. Reflection and focusing. In Figure 6, we show an axisymmetric calculation of the ESWT wave propagation and focusing in water alone, in a domain bounded by the ellipsoidal reflector of the Dornier HM3. Figure 6, top left, shows the initial spherical propagation of the pressure wave, as well as the grids where the calculation is being refined. The grid must be refined around the pressure wave as well as the reflector. Figure 6, top right and bottom left, shows the propagation of the wave and evolution of the adaptive grid structures. At later times the grid is only being refined near the pressure wave. The sharp results and absence of spurious oscillations in the pressure measurement at F2 indicate that AMR together with our Cartesian grid approach enables us to capture the reflection at the interface.
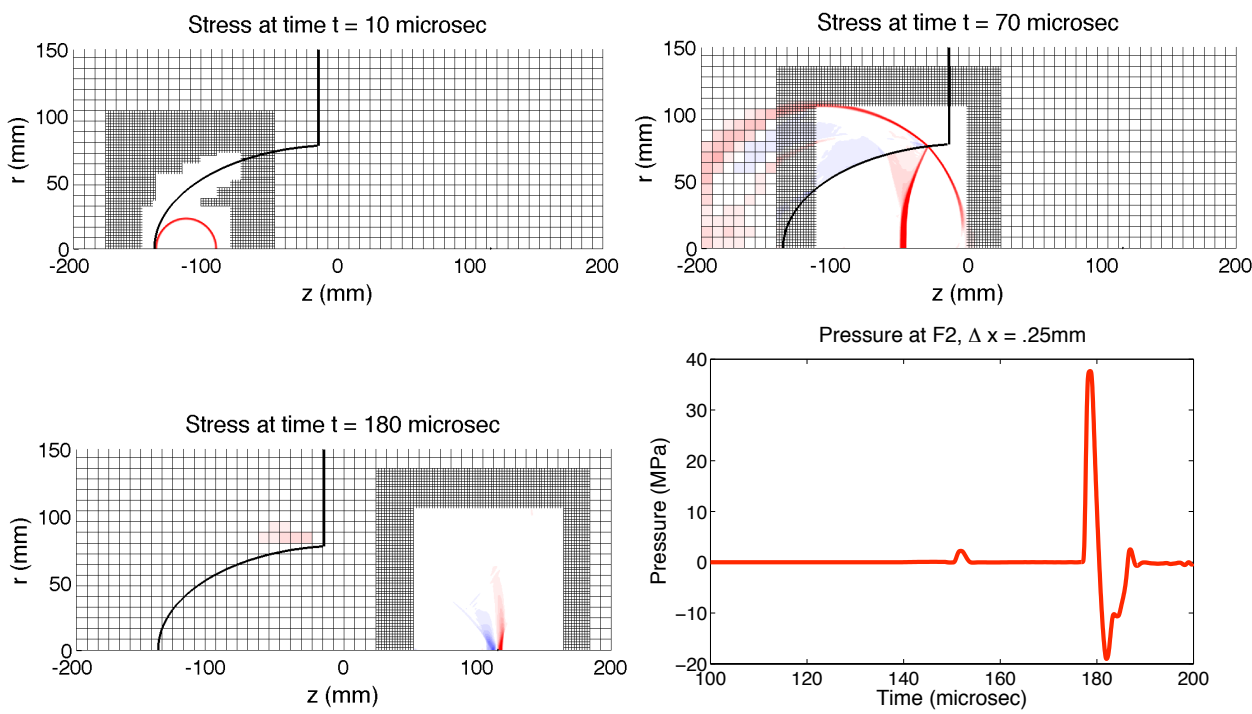

Figure 6. Axisymmetric calculation of the pressure pulse generated by a spherical highpressure bubble centered $z=-115$ (the focus F1 of the ellipsoidal reflector). Three levels of AMR are used and grid lines are shown only on levels 1 and 2. The level-3 grid has a resolution of $\Delta z=\Delta r=0.25 \mathrm{~mm}$. Top left: at $t=10$ the pulse has nearly reached the reflector. Top right: at $t=70$ the incident, transmitted, and reflected pulses are visible. Bottom left: at $t=180$ the reflected pulse has focused near $z=115$ (the focus F2). Bottom right: the time history of the pressure at F2. The direct (unreflected) wave passes F2 at $t \approx 150$ and the focused pulse arrives at $t \approx 180$. 
5.2. Axisymmetric sphere. We used an axisymmetric test problem in order to compare the solutions obtained with the two-dimensional and three-dimensional codes. The initial condition for this experiment was an analytic form for an ESWT pressure wave used in [49]. In the two-dimensional case, we specified the pressure as a function of the radial distance from $\mathrm{F} 1(-115,0)$. In the three-dimensional case, we rotated the same two-dimensional initial condition about the $x$-axis. The grid resolution was $\Delta x=0.25 \mathrm{~mm}$.

Results with contour lines are shown in Figure 7. The maximum values in each of the three cases are nearly the same, but there are slight discrepancies in the contour
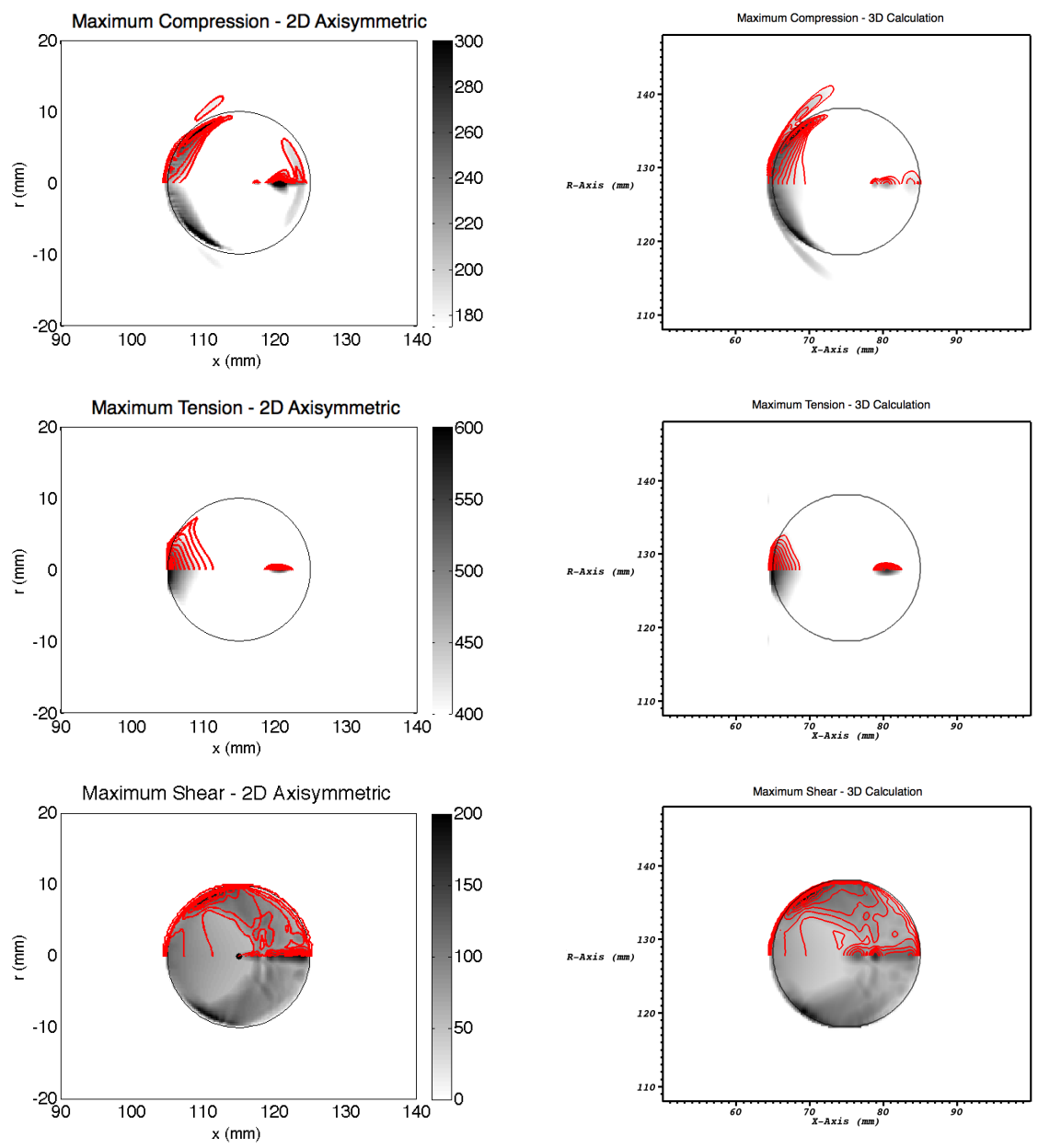

Figure 7. Results from calculation of a shockwave interacting with an acrylic sphere. The left column shows two-dimensional axisymmetric results and the right column shows a corresponding cross section of full three-dimensional calculation. Top: maximum compression; middle: maximum tension; bottom: maximum shear. 


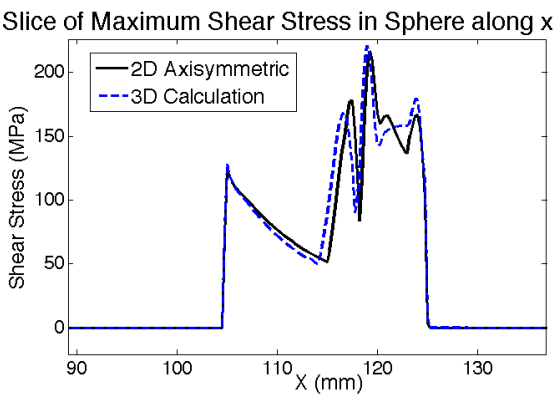

Figure 8. Comparison of maximum shear stress from two-dimensional and threedimensional calculations as a function of $x$ along $y=z=0$. The difference in the results is likely caused by averaging of the initial condition onto the three-dimensional domain and the boundary conditions on the axisymmetric calculation at $r=0$, but the two calculations predict comparable location and magnitude of maximal shear stress deposition.

lines. Figure 8 shows a one-dimensional slice of the maximum shear calculation in the two-dimensional and three-dimensional codes, which makes it clear that the peak of maximal shear stress is in the same location and has the same value. The general shape of the maximum stress deposition pattern are similar in both cases. The difference in the two solutions is likely caused by the solid wall boundary condition that is used at $r=0$. Only waves that are propagating normal to that boundary are perfectly reflected, otherwise some error is generated.

5.3. Nonunions. ESWT has recently been used for the treatment of nonunions or bone fractures that fail to heal [9]. One question that is of interest to clinicians is whether or not the angle of treatment has an effect on healing. We assume that healing is related to the magnitude of stress applied near the treatment area, although the connection between the applied force and biological response is not yet understood. In the fluid there is no shear stress. However, at the liquid-solid interfaces, shear stresses are generated by the shockwave and stimulate motion both at the surface and within the material. The motion of the biological materials (e.g., the periosteum, interstitial fluid, mechanotransduction) is likely to be important in the healing process $[25 ; 44 ; 56 ; 13 ; 27 ; 43 ; 53]$, and modeling the magnitude and location of the stress deposition is a good first step toward understanding the shear and tensile displacements caused by ESWT. We should stress, however, that the healing mechanisms are not well understood and we are not claiming that magnitude of the applied stress is the most important or only biological mechanism involved in the healing process. As mentioned in Section 1, several studies have indicated that cyclic application of mechanical loading leads to the generation of new bone. The work of Isaksson et al. [27], indicates that the most accurate predictors for bone healing are those based on shear strain and fluid flow, however, there is no 

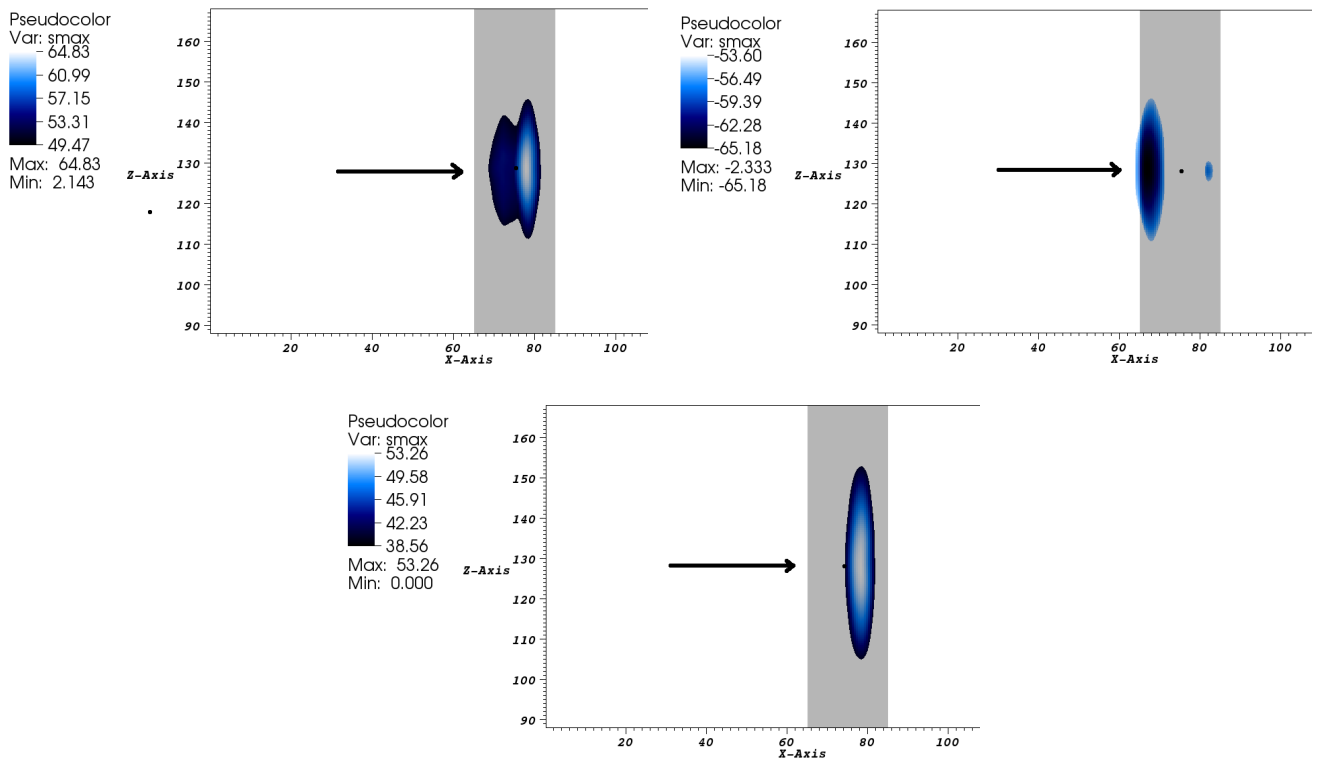

Figure 9. Three-dimensional results for the direct treatment of a complete cylinder. This figure shows two-dimensional slices of maximum compression, tension and shear along $y=0$ for treatment where the ESWT wave propagates along the $x$-axis, as indicated by the arrow. The dot illustrates the location of F2.

single model that can predict all features of the healing process, so more work is necessary [39].

In an actual treatment, the clinician generally sets up the device so that the focus is aligned with the ailment. For example, in the case of a broken bone, the clinician will set up the device so that F2 is in the center of the break. However, given the heterogeneous media, it is not clear that the maximal stresses will actually be observed at F2, as would be expected in pure water. We used our model to investigate the location of maximal stress deposition relative to F2. In these calculations we considered two different geometries, a complete cylinder, representing the long shaft of a healthy bone, and a broken cylinder, representing a nonunion. The results from calculations where the idealized bone was perpendicular to the direction of the pressure wave front are shown in Figures 9 and 10. We found that the break has a significant impact on the location of stress deposition.

We used these geometries to perform a variety of experiments. We rotated the direction of treatment by 45 and 60 degrees relative to the $x$-axis and calculated both the magnitude of the maximum compressive, tensile and shear stresses, as well as the distance from the focus $\mathrm{F} 2$ of the device.

In the case of the broken cylinder, the maximum stress deposition in the direct experiment is similar to that of the unbroken cylinder, except that the there are two 

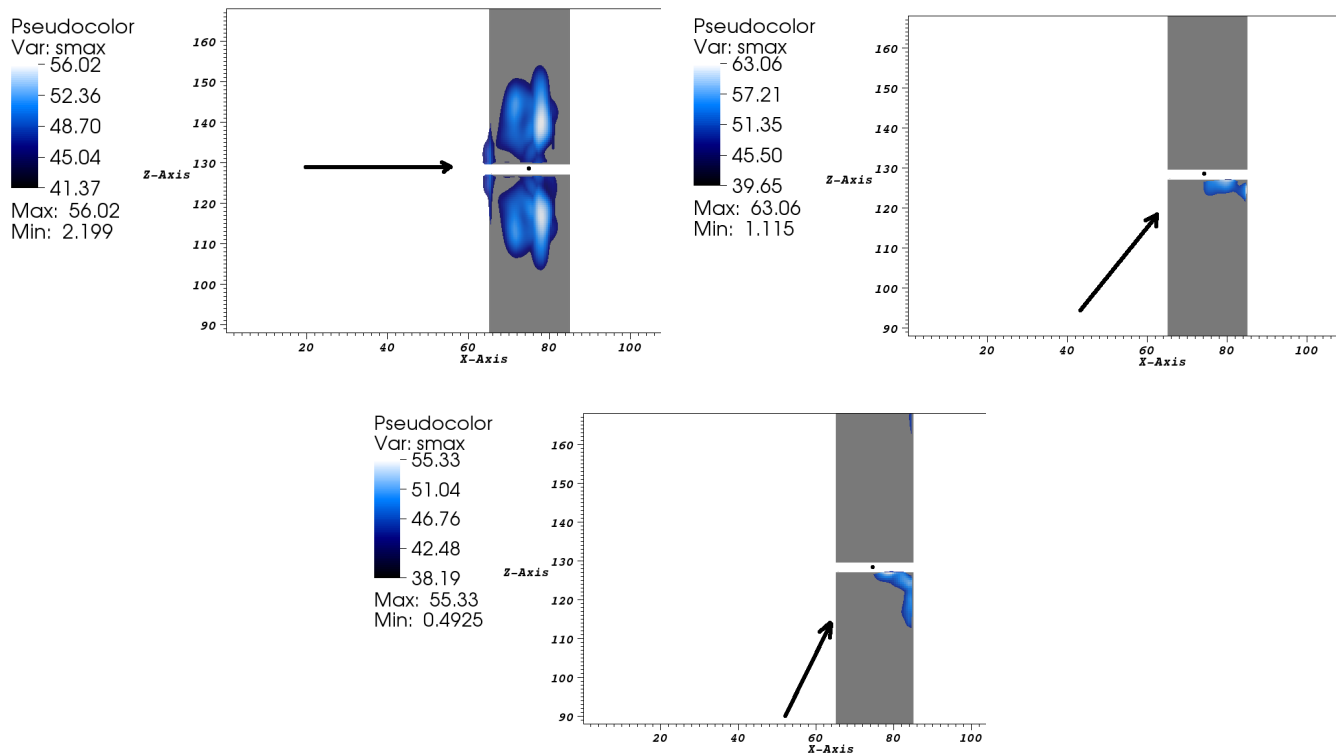

Figure 10. Three-dimensional results for the direct treatment of a broken cylinder. This figure shows two-dimensional slices of maximum compression along $y=0$ for treatment along the $x$-axis, 45 -degree rotation and 60-degree rotation about the $y$-axis. The arrows indicate the angle of treatment in each case and the dot illustrates the location of F2.

locations of maximal stress deposition on either side of the break. The pressures in the bone are larger than in the fluid due to reflection at the fluid-solid interface, so the contours of maximum stress are concentrated on either side of the gap. The location along the $x$-axis is nearly the same as in the unbroken cylinder, and the distances from the ideal focal point, $\mathrm{F} 2$, are also similar.

As the angle of treatment is varied, there is less of a shift in the $z$ direction for the shear and compressive stresses. This is caused by the impedance difference between the fluid and solid material at the gap, which is located close to F2. If the gap were shifted along the $z$-axis from the focal point, there would be a corresponding shift in the location of maximum shear and compression. Geometrically, the shape of the regions of compressive and shear stress are quite different from the direct case. Instead of being an ellipsoidal shape, the regions are compressed into the corner of the lower-half of the cylinder. Again, this is caused by the impedance jump at the fluid-solid interface. The region of maximum tension deposition is similar to that of the unbroken cylinder case, though it is also affected by the gap and the tension is concentrated on the upper half of the cylinder.

It is clear from the literature $[43 ; 44 ; 30 ; 13 ; 27 ; 11 ; 23]$, that mechanical loading is important in bone healing. The implication of our computational experiments is that the angle of treatment will affect stress deposition and therefore may be 

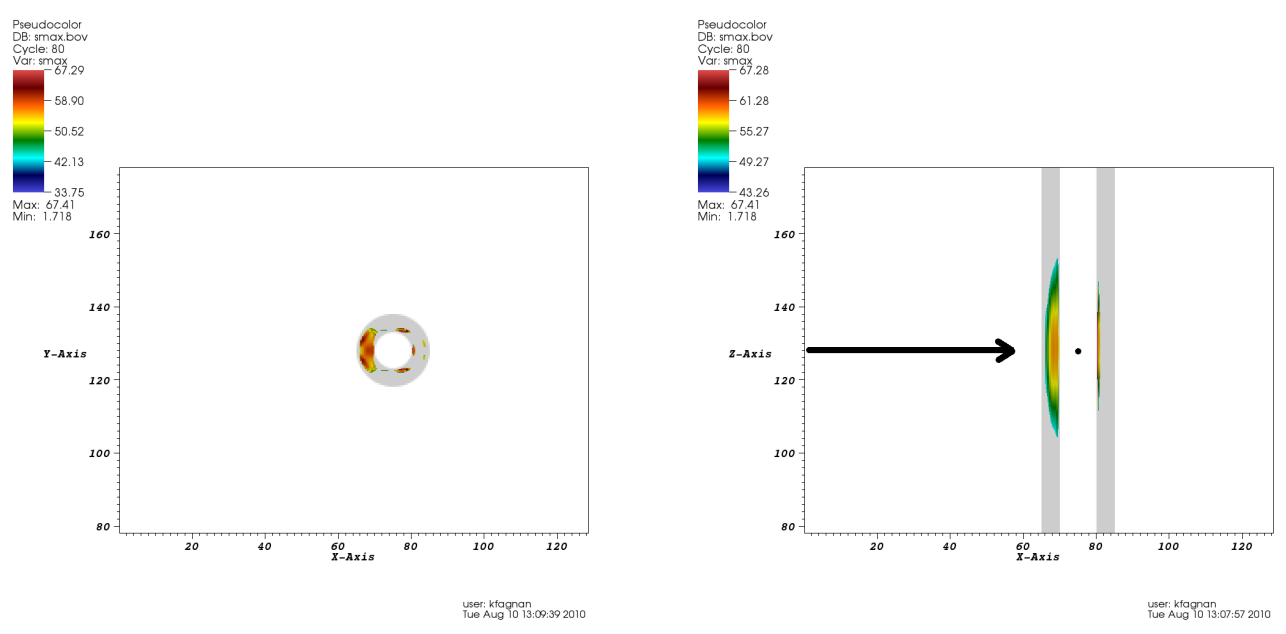

Figure 11. An additional interface calculation showing the more realistic treatment of a cylinder with a fluid-filled cavity. Left: a slice along $z=0$ showing the concentration of stress in the front of the idealized bone, with additional smaller pockets of maximum stress due to reflection in the back half of the bone. Right: a slice along $y=0$ which further demonstrates the stress concentration in the first half of the bone. The arrow in the figure at right indicates the direction of ESWT wave propagation and the dot indicates the location of F2.

important in treatment optimization. For example, in order to maximize shear stress at the tissue-bone interface, our preliminary computations indicate that it might be best to treat the patient at an oblique angle. However, if the goal is to maximize shear stress in the gap of the broken bone, then treating the patient at a 90-degree angle may be better than treating at either the 45- or 60-degree angle. We stress however that the biological mechanisms must be better understood and more experiments must be done in conjunction with laboratory and clinical treatments before these calculations could be used to make specific clinical recommendations.

In Figure 11 we show two-dimensional slices of a calculation with a more realistic, but still idealized, long bone geometry. In a the shaft of a long bone, there is a marrow-filled canal running through the center. Marrow is typically modeled as a viscoelastic material [37], but for this first approximation we just used a fluidfilled canal. The impedance difference in the two materials is similar and therefore illustrates the behavior that we are interested in, the change in maximal stress deposition. In contrast to the solid cylinder above, the contours of maximal stress are concentrated in the front side of the hollow cylinder. Figure 11, right, shows that there are also two regions of additional stress concentration in the backside of the hollow cylinder. This example highlights the importance in understanding where these impedance jumps occur in order to optimally treat the patient. 
5.4. Heterotopic ossification. A heterotopic ossification (HO) is a growth of bonelike material in soft tissue. HOs often grow spontaneously in tissue that has been traumatized due to injury or amputation. An example of an $\mathrm{HO}$ is shown in Figure 12, left, which shows the pelvis and an HO, using data extracted from a patient's CT scan. In this case, the HO has grown around the right hip joint and is inhibiting the patient's range of motion. The goal of the HO treatment is not to pulverize the ossification, but to break up the adhesion between the $\mathrm{HO}$ and the joint, in order to restore the patient's range of motion. There is no clear division between the $\mathrm{HO}$ and bone in the CT scan because both are composed of materials that have similar densities. However, the HO does not have the same woven structure that is present in bone, so the two will likely have different material properties, even though the densities are similar. This similarity means that we are uncertain as to how strong the connection or adhesion is between the $\mathrm{HO}$ and the bone, which will directly impact the number of shocks needed to restore the patient's range of motion.

We are able to use our model to investigate the effect of the angle of treatment on the observed stresses in the region near the HO. Since the composition and material properties of the ossification are not well understood, we can also use the model to vary the material properties of the ossification and investigate the sensitivity of the results to these parameters. We found that both the strength of the connection between the $\mathrm{HO}$ and bone, as well as the composition of the $\mathrm{HO}$, had a significant effect on the location of maximum stress in the object [18].

It is challenging to infer anything meaningful from the images in the full threedimensional calculation in Figure 12, left, so we have also included a two-dimensional slice of the maximum shear in Figure 12, right. Here the gray regions
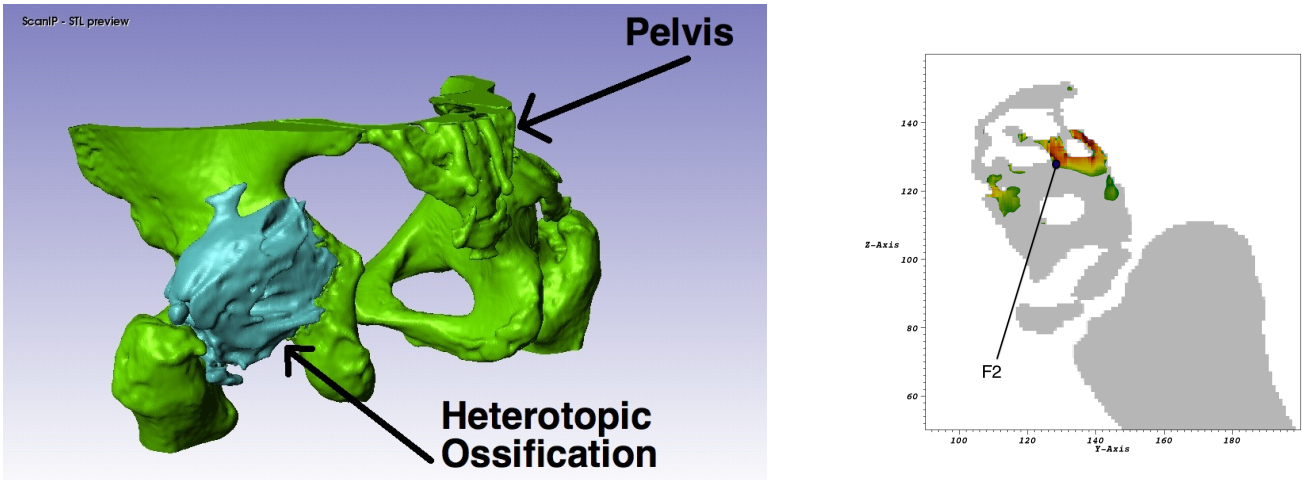

Figure 12. Left: the three-dimensional CT patient data illustrating the heterotopic ossification (blue) attached to the right hip joint (green). Right: a slice at $x=115$ of the two-dimensional calculation shows how the pockets of fluid lead to stress concentration in the substructure of the ossification, the dot indicates the location of F2 and the direction of treatment is into the page. 
represent the bone-like $\mathrm{HO}$ material and we assume any gaps are filled with fluid. It is clear that the interior of the ossification is complex and contains many fluid-filled pockets that affect, in this case, the location of the maximum shear stress.

Given the complex nature of the $\mathrm{HO}$ and subsequent difficulty interpreting the three-dimensional results, we have also used an idealized ossification to investigate some facets of the shockwave interaction with the varying material properties. One example of this is shown in Figure 13, where we have simulated a case where the ossification (the crescent in the two-dimensional images) is not strongly attached to the bone (the cylinder) and calculated the maximal shear stress as a result of two different treatment angles. Figure 13, left, illustrates the result when the ESWT device is aimed orthogonal to the gap between the $\mathrm{HO}$ and the cylinder. Figure 13, right, is the result when the device is aimed so the shockwaves propagate parallel to the gap between the $\mathrm{HO}$ and bone. It has been indicated that maximum shear stress is important in causing the $\mathrm{HO}$ to break, so it is desirable to deposit the maximum amount of shear as close to the $\mathrm{HO} /$ bone interface as possible. In this case, it is better to treat the HO in the direction indicated in Figure 13, right, since the shear stress is concentrated along the gap.

According to our computational results, the pockets of fluid within an $\mathrm{HO}$ and strength of adhesion to the bone surface will affect the stress deposition and therefore the location of the eventual break in the ossification. Further investigation is required to be conclusive, but our results indicate that if the fluid pockets are in the propagation path of the shock wave, they may cause the maximum stresses to occur away from the adhesion site, making the treatment less effective. In reality, the composition of the HO is unknown and we do not have a good characterization for the material properties of the ossifications. However, the strong impedance mismatch between fluid and bone, as well as the inability of the fluid to support shear stress, indicate that the presence of fluid-filled pockets will have an effect on the stress deposition. We should note here that our modeling work does not take into account the propagation of successive shocks or failure models within the material, which should ultimately be incorporated in order to the determine the optimal treatment. This is an area for future work.

\section{Conclusion}

In this paper we have proposed a new model for ESWT. We have demonstrated that the Tait equation of state is sufficient for the pressures that arise in ESWT, that enables us to drop the energy equation from our model. We have shown that the fluid and solid can be modeled with the same set of Lagrangian equations since the particle displacements are small. This approach allowed us to utilize existing numerical methodology, consisting of high-resolution shock-capturing methods 

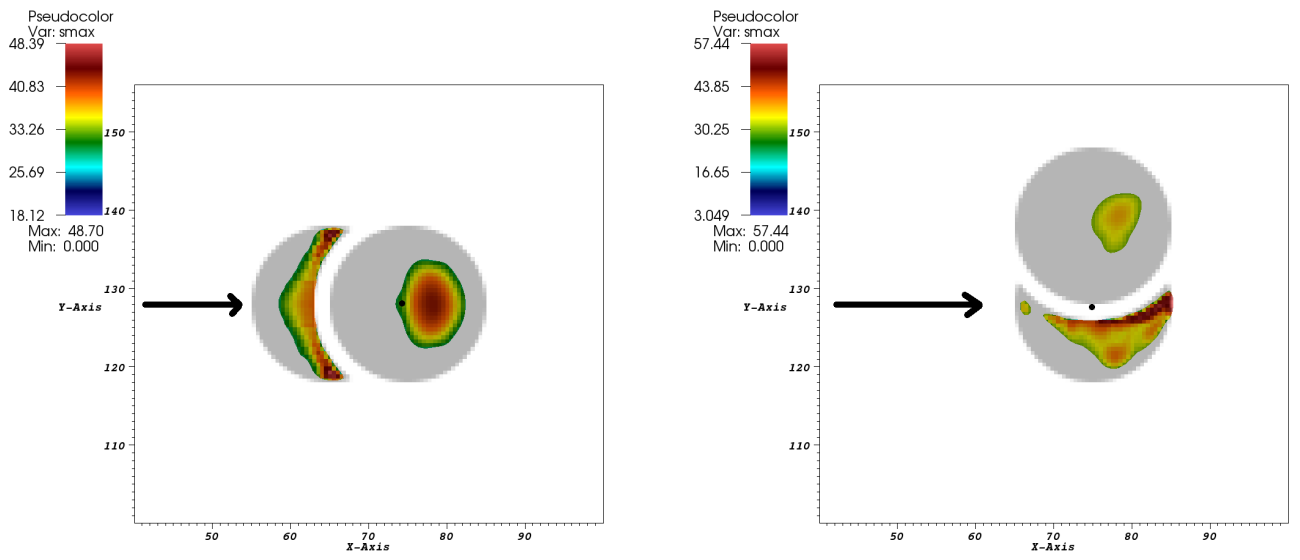

Figure 13. Calculations for an idealized ossification that demonstrates the difference in shear stress deposition when treating the HO from different directions. Since the goal is to disrupt the $\mathrm{HO}$ at the interface between the bone and the $\mathrm{HO}$, the figure on the right indicates that it would be optimal send shock waves parallel to the break, instead of perpendicular to it. The arrows indicate the direction of ESWT propagation and the dots indicate the location of F2.

together with adaptive mesh refinement, to efficiently calculate solutions to these equations for a variety of idealized biological problems. We have also demonstrated that we can effectively handle interfaces between different materials on Cartesian grids. Using this methodology we were able to explore, even in geometrically complicated structures, how the interfaces between the fluid and solid materials affect the distribution of maximal stress in several problems of clinical interest. We should note that the models for the biological materials are idealized, so it is difficult to extrapolate from these experiments to reality without conducting further experiments.

Maximizing stress in specific regions seems important in both the healing and destruction of biological tissues. Shear stress is thought to be play a role in the stimulation of biological tissues [54; 25; 20;23; 13; 30; 43]. Mechanical loading is thought to play a role in the formation of bone tissue, and as discussed in Section 1, shear and compressive displacements generated by loading influence bone healing $[43 ; 44 ; 30 ; 13 ; 27 ; 11 ; 23]$. Shear stress is also important in predicting the break up of kidney stones [49].

The model we have developed has been used to investigate idealized nonunions and heterotopic ossifications, and we have shown a few examples to illustrate this. A broader range of calculations are available in [18]. 
The focus of this paper has been the effect that material interfaces between tissue and bone have on the transmission, reflection, and focusing of the shock wave. Very simple models have been used for the material on each side of the interface: compressible fluid with a Tait equation of state in the tissue and linear isotropic elasticity in the bone. We believe that this level of macroscopic modeling can already reveal interesting features of the stress that may be clinically important. In particular, focusing may occur in regions displaced from where it would be observed in pure water, and mode conversion at an interface can generate shear waves in the bone that are not present in the focusing shock wave in fluid.

To consider the effect of stress on individual osteocytes, a much more detailed model would be necessary that is beyond the scope of this work. In particular, this would require modeling the microscale fluid-filled canaliculi within the bone through which the osteocyte processes extend. Work is currently underway in this direction, and also on intermediate levels of modeling in which the bone is modeled as an orthotropic poroelastic material. These equations can be solved with essentially the same high resolution finite volume methods used here, after implementing a more complicated Riemann solver [32;33], and with the same software for adaptive mesh refinement. Another possible extension is to investigate viscoelastic tissue models that may be superior to the Tait equation for water that is currently used.

\section{Acknowledgements}

This work was supported in part by NIH Grant 5R01AR53652-2, NSF Grants DMS0609661 and DMS-0914942, and the Founders Term Professorship in Applied Mathematics at the University of Washington. The authors would like to thank Donna Calhoun and the ANAG group at Lawrence Berkeley Laboratory for their assistance with the ChomboClaw calculations. This research was supported in part by the National Science Foundation through TeraGrid resources provided by TACC under grant number TG-DMS090036T.

\section{References}

[1] Chombo: software for adaptive solutions of partial differential equations, webpage, Applied Numerical Algorithms Group (ANAG), Lawrence Berkeley National Laboratory, 2009.

[2] P. Augat, J. Merk, S. Wolf, and L. E. Claes, Mechanical stimulation by external application of cyclic tensile strains does not effectively enhance bone healing, Journal of Orthopaedic Trauma 15 (2001), 54-60.

[3] M. Averkiou and R. Cleveland, Modeling of an electrohydraulic lithotripter with the KZK equation, Journal for the Acoustical Society of America 106 (1999), no. 1, 102-112. 
[4] D. S. Bale, R. J. Leveque, S. Mitran, and J. A. Rossmanith, A wave propagation method for conservation laws and balance laws with spatially varying flux functions, SIAM J. Sci. Comput. 24 (2002), no. 3, 955-978. MR 2004a:65098

[5] M. J. Berger and P. Colella, Local adaptive mesh refinement for shock hydrodynamics, J. Comput. Phys. 82 (1989), 64-84.

[6] M. J. Berger and I. Rigoutsos, An algorithm for point clustering and grid generation, IEEE Trans. Sys. Man \& Cyber. 21 (1991), 1278-1286.

[7] M. J. Berger and R. J. Leveque, Adaptive mesh refinement using wave-propagation algorithms for hyperbolic systems, SIAM J. Numer. Anal. 35 (1998), no. 6, 2298-2316. MR 2000c:65082

[8] M. J. Berger and J. Oliger, Adaptive mesh refinement for hyperbolic partial differential equations, J. Comput. Phys. 53 (1984), no. 3, 484-512. MR 85h:65211

[9] R. Biedermann, A. Martin, G. Handle, T. Auckenthaler, C. Bach, and M. Krismer, Extracorporeal shock waves in the treatment of nonunions, Journal of Trauma 54 (2003), no. 5, 936-42.

[10] D. A. Calhoun, P. Colella, and R. J. LeVeque, CHOмBO-CLAW software, web site.

[11] D. R. Carter, G. S. Beaupre, N. J. Giori, and J. A. Helms, Mechanobiology of skeletal regeneration, Clinical Orthopaedics and Related Research 355 (Suppl) (1998), S41-55.

[12] T. Christopher, Modeling the Dornier HM3 lithotripter, Journal of the Acoustical Society of America 96 (1994), no. 5, 3088-3095.

[13] L. E. Claes and C. A. Heigele, Magnitudes of local stress and strain along osseous surfaces predict the course and type of fracture-healing, The Journal of Biomechanics 32 (1999), 255266.

[14] L. E. Claes, H. J. Wilke, P. Augat, S. Rubenacker, and K. J. Margevicius, Effect of dynamization on gap healing of diaphyseal fractures under external fixation, Cinical Biomechanics 10 (1995), 227-234.

[15] Clawpack Team, CLAWPACK software, web page, 2013.

[16] R. O. Cleveland and O. Sapozhnikov, Modeling elastic wave propagation in kidney stones with application to shock wave lithotripsy, Journal of the Acoustical Society of America 118 (2005), no. 4, 2667-2676.

[17] A. J. Coleman, J. Saunders, R. Preston, and D. Bacon, Pressure waveforms generated by a Dornier extra-corporeal shock-wave lithotripter, Ultrasound in Medicine and Biology 13 (1987), 651-657.

[18] K. M. Fagnan, High-resolution finite volume methods for extracorporeal shock wave therapy, Ph.D. thesis, University of Washington, 2010. MR 2941302

[19] K. Fagnan, R. J. LeVeque, T. J. Matula, and B. MacConaghy, High-resolution finite volume methods for extracorporeal shock wave therapy, Hyperbolic problems: theory, numerics, applications (S. Benzoni-Gavage and D. Serre, eds.), Springer, Berlin, 2008, pp. 503-510. MR 2549183

[20] J. Freund, T. Colonius, and A. Evan, A cumulative shear mechanism for tissue damage initiation in shock-wave lithotripsy, Ultrasound in Medicine and Biology 33 (2007), 1495-1503.

[21] Y. C. Fung, Biomechanics: mechanical properties of living tissues, Springer, 1993.

[22] E. Garner, R. Lakes, T. Lee, C. Swan, and R. Brand, Viscoelastic dissipation in compact bone: implications for stress-induced fluid flow in bone, Journal of Biomechanical Engineering 122 (2000), 166-73. 
[23] A. E. Goodship and J. Kenwright, The influence of induced micromovement on the healing of experimental tibial fractures, Journal of Bone and Joint Surgery British Volume 67 (1985), 650-655.

[24] M. Hamilton, Transient axial solution for the reflection of a spherical wave from a concave ellipsoidal mirror, Journal of the Acoustical Society of America 93 (1993), no. 3, 1256-1266.

[25] M. V. Hillsley and J. A. Frangos, Review of "Bone tissue engineering: the role of interstitial fluid flow”, Biotechnology and Bioengineering 43 (1994), 573-581.

[26] C. Huang and R. Ogawa, Mechanotransduction in bone repair and regeneration, The FASEB Journal 23 (2010), 3625-3632.

[27] H. Isaksson, W. Wilson, C. C. van Donkelaar, R. Huiskes, and K. Ito, Comparison of biophysical stimuli for mechano-regulation of tissue differentiation during fracture-healing, The Journal of Biomechanics 39 (2006), 1507-1516.

[28] M. J. Ivings, D. M. Causon, and E. F. Toro, On Riemann solvers for compressible liquids, Internat. J. Numer. Methods Fluids 28 (1998), no. 3, 395-418. MR 99e:76088

[29] T. Keaveny, X. E. Guo, E. F. Wachtel, T. A. McMahon, and W. C. Hayes, Trabecular bone exhibits fully linear elastic behavior and yields at low strains, Journal of Biomechanics 27 (1994), 1127-1129, 1131-1136.

[30] D. Lacroix and P. J. Prendergast, A mechano-regulation model for tissue differentiation during fracture-healing: analysis of gap size and loading, The Journal of Biomechanics 35 (2002), 1163-1171.

[31] J. O. Langseth and R. J. LeVeque, A wave propagation method for three-dimensional hyperbolic conservation laws, J. Comput. Phys. 165 (2000), no. 1, 126-166. MR 2001i:65110

[32] G. I. Lemoine, Numerical modeling of poroelastic-fluid systems using high-resolution finite volume methods, Ph.D. thesis, University of Washington, 2013.

[33] G. I. Lemoine, M. Y. Ou, and R. J. LeVeque, High-resolution finite volume modeling of wave propagation in orthotropic poroelastic media, SIAM J. Sci. Comput. 35 (2013), no. 1, B176B206. MR 3033065

[34] R. J. LeVeque, Wave propagation algorithms for multi-dimensional hyperbolic systems, J. Comput. Phys. 131 (1997), 327-353.

[35] _ Finite volume methods for hyperbolic problems, Cambridge Texts in Applied Mathematics, no. 31, Cambridge University Press, 2002. MR 2003h:65001

[36] _ Finite-volume methods for non-linear elasticity in heterogeneous media, Internat. J. Numer. Methods Fluids 40 (2002), no. 1-2, 93-104. MR 2003h:74078

[37] R. B. Martin, D. B. Burr, and N. A. Sharkey, Skeletal tissue mechanics, Springer, New York, 1998.

[38] T. J. Matula, P. R. Hilmo, and M. R. Bailey, A suppressor to prevent direct wave-induced cavitation in shock wave therapy devices, Journal of the Acoustical Society of America 118 (2005), no. 1, 178-185.

[39] E. F. Morgan, R. E. Gleason, L. N. M. Hayward, P. L. Leong, and K. T. Salisbury-Paolomares, Mechanotransduction and fracture repair, Journal of Bone and Joint Surgery American Volume 90 (Suppl 1) (2008), 25-30.

[40] G. Mouzopoulos, M. Stamatakos, D. Mouzopoulos, and M. Tzurbakis, Extracorporeal shock wave treatment for shoulder calcific tendonitis: a systematic review, Skeletal Radiology 36 (2008), no. 9, 803-811. 
[41] M. Nakahara, K. Nagayama, and Y. Mori, Shockwave dynamics of high pressure pulse in water and other biological materials based on Hugoniot data, Japanese Journal of Applied Physics 47 (2008), 3510.

[42] J. A. Ogden, A. Toth-Kischkat, and R. Schultheiss, Principles of shock wave therapy, Clinical Orthopaedics and Related Research 387 (2001), 8-17.

[43] S. H. Park, K. O'Connor, H. McKellop, and A. Sarmiento, The influence of active shear or compressive motion on fracture-healing, The Journal of Bone and Joint Surgery American Volume 80 (1998), 868-878.

[44] P. J. Prendergast, R. Huiskes, and K. Soballe, Biophysical stimuli on cells during tissue differentiation at implant interfaces, The Journal of Biomechanics 30 (1997), 539-548.

[45] A. G. Robling, F. M. Hinant, D. B. Burr, and C. H. Turner, Improved bone structure and strength after long-term mechanical loading is greatest if loading is separated into short bouts, Journal of Bone Mineral Research 17 (2002), 1545-1554.

[46] _ Shorter, more frequent mechanical loading sessions enhance bone mass, Medicine and Science in Sports and Exercise 34 (2002), 196-202.

[47] T. Saito, M. Marumoto, H. Yamashita, S. H. R. Hosseini, A. Nakagawa, T. Hirano, and K. Takayama, Experimental and numerical studies of underwater shock wave attenuation, Shock Waves 13 (2003), 139-148.

[48] O. Sapozhnikov, M. Bailey, and R. O. Cleveland, The role of shear and longitudinal waves in the kidney stone comminution by a lithotripter shock pulse, Journal of the Acoustical Society of America 115 (2004), 2562.

[49] O. Sapozhnikov, A. D. Maxwell, B. MacConaghy, and M. Bailey, A mechanistic analysis of stone fracture in lithotripsy, Journal of the Acoustical Society of America 121 (2007), no. 2, $1190-1202$.

[50] L. Saxon, A. Robling, I. Alam, and C. Turner, Mechanosensitivity of the rat skeleton decreases after a long period of loading, but is improved with time off, Bone 36 (2005), 454-464.

[51] M. Tanguay, Computation of bubbly cavitating flow in shock wave lithotripsy, Ph.D. thesis, California Institute of Technology, 2004.

[52] W. R. Taylor, E. Roland, H. Ploeg, D. Hertig, R. Klabunde, M. D. Warner, M. C. Hobatho, L. Rakotomanana, and S. E. Clift, Determination of orthotropic bone elastic constants using FEA and modal analysis, Journal of Biomechanics 35 (2002), 767-773.

[53] C. H. Turner and F. M. Pavalko, Mechanotransduction and functional response of the skeleton to physical stress: the mechanisms and mechanics of bone adaptation, Journal of Orthopaedic Science 3 (1998), 346-355.

[54] C.-J. Wang, K.-E. Huang, Y.-C. Sun, Y.-J. Yang, J.-Y. Ko, L.-H. Weng, and F.-S. Wang, VEGF modulates angiogenesis and osteogenesis in shockwave-promoted fracture healing in rabbits, Journal of Surgical Research 171 (2011), no. 1, 114-119.

[55] C.-J. Wang, F.-S. Wang, J.-Y. Ko, H.-Y. Huang, C.-J. Chen, Y.-C. Sun, and Y.-J. Yang, Extracorporeal shockwave therapy shows regeneration in hip necrosis, Rheumatology 47 (2008), no. 4, 542-546.

[56] S. Weinbaum, S. Cowin, and Y. Zeng, A model for the excitation of osteocytes by mechanical loading-induced bone fluid shear stress, Journal of Biomechanics 27 (1994), 339-360.

Received December 6, 2010. 
KIRSTEN FAGNAN: kmfagnan@lbl.gov

NERSC/JGI, Lawrence Berkeley National Laboratory, 1 Cyclotron Road, MS 943R0256, Berkeley, CA 94720, United States

RANDALL J. LEVEQUE: rj1@amath. washington. edu Department of Applied Mathematics, University of Washington, Box 352420, Seattle, WA 98195-2420, United States

Thomas J. MatUla: matula@apl . washington .edu Applied Physics Laboratory, University of Washington, 1013 NE 40th Street, Box 355640, Seattle, WA 98105-6698, United States 


\title{
Communications in Applied Mathematics and Computational Science
}

\author{
msp.org/camcos
}

EDITORS

MANAGING EDITOR

John B. Bell

Lawrence Berkeley National Laboratory, USA

jbbell@lbl.gov

\section{BOARD OF EDITORS}

\begin{tabular}{|c|c|c|c|}
\hline Marsha Berger & $\begin{array}{l}\text { New York University } \\
\text { berger@cs.nyu.edu }\end{array}$ & Ahmed Ghoniem & $\begin{array}{l}\text { Massachusetts Inst. of Technology, USA } \\
\text { ghoniem@mit.edu }\end{array}$ \\
\hline Alexandre Chorin & $\begin{array}{l}\text { University of California, Berkeley, USA } \\
\text { chorin@math.berkeley.edu }\end{array}$ & Raz Kupferman & $\begin{array}{l}\text { The Hebrew University, Israel } \\
\text { raz@math.huji.ac.il }\end{array}$ \\
\hline Phil Colella & $\begin{array}{l}\text { Lawrence Berkeley Nat. Lab., USA } \\
\text { pcolella@lbl.gov }\end{array}$ & Randall J. LeVeque & $\begin{array}{l}\text { University of Washington, USA } \\
\text { rj1@amath.washington.edu }\end{array}$ \\
\hline Peter Constantin & $\begin{array}{l}\text { University of Chicago, USA } \\
\text { const@cs.uchicago.edu }\end{array}$ & Mitchell Luskin & $\begin{array}{l}\text { University of Minnesota, USA } \\
\text { luskin@umn.edu }\end{array}$ \\
\hline Maksymilian Dryja & $\begin{array}{l}\text { Warsaw University, Poland } \\
\text { maksymilian.dryja@acn.waw.pl }\end{array}$ & Yvon Maday & $\begin{array}{l}\text { Université Pierre et Marie Curie, France } \\
\text { maday@ann.jussieu.fr }\end{array}$ \\
\hline M. Gregory Forest & $\begin{array}{l}\text { University of North Carolina, USA } \\
\text { forest@amath.unc.edu }\end{array}$ & James Sethian & $\begin{array}{l}\text { University of California, Berkeley, USA } \\
\text { sethian@ math.berkeley.edu }\end{array}$ \\
\hline Leslie Greengard & $\begin{array}{l}\text { New York University, USA } \\
\text { greengard@cims.nyu.edu }\end{array}$ & Juan Luis Vázquez & $\begin{array}{l}\text { Universidad Autónoma de Madrid, Spain } \\
\text { juanluis.vazquez@uam.es }\end{array}$ \\
\hline Rupert Klein & $\begin{array}{l}\text { Freie Universität Berlin, Germany } \\
\text { rupert.klein@pik-potsdam.de }\end{array}$ & Alfio Quarteroni & $\begin{array}{l}\text { Ecole Polytech. Féd. Lausanne, Switzerland } \\
\text { alfio.quarteroni@epfl.ch }\end{array}$ \\
\hline \multirow[t]{2}{*}{ Nigel Goldenfeld } & $\begin{array}{l}\text { University of Illinois, USA } \\
\text { nigel@uiuc.edu }\end{array}$ & Eitan Tadmor & $\begin{array}{l}\text { University of Maryland, USA } \\
\text { etadmor@cscamm.umd.edu }\end{array}$ \\
\hline & & Denis Talay & $\begin{array}{l}\text { INRIA, France } \\
\text { denis.talay@inria.fr }\end{array}$ \\
\hline
\end{tabular}

\section{PRODUCTION}

production@msp.org

Silvio Levy, Scientific Editor

See inside back cover or msp.org/camcos for submission instructions.

The subscription price for 2013 is US \$75/year for the electronic version, and \$105/year ( $\$ 15$, if shipping outside the US) for print and electronic. Subscriptions, requests for back issues from the last three years and changes of subscribers address should be sent to MSP.

Communications in Applied Mathematics and Computational Science (ISSN 2157-5452 electronic, 1559-3940 printed) at Mathematical Sciences Publishers, 798 Evans Hall \#3840, c/o University of California, Berkeley, CA 94720-3840, is published continuously online. Periodical rate postage paid at Berkeley, CA 94704, and additional mailing offices.

CAMCoS peer review and production are managed by EditFLOW ${ }^{\circledR}$ from Mathematical Sciences Publishers.

\section{PUBLISHED BY}

mathematical sciences publishers

nonprofit scientific publishing

http://msp.org/

(C) 2013 Mathematical Sciences Publishers 


\section{Communications in Applied Mathematics and Computational Science}

vol. 8

no. 1

2013

On the origin of divergence errors in MHD simulations and consequences for $\quad I$ numerical schemes

FRIEDEMANN KEMM

Renormalized reduced models for singular PDEs

PANOS StINIS

Legendre spectral-collocation method for Volterra integral differential equations with nonvanishing delay

YANPING Chen and ZHendong GU

A cartesian grid embedded boundary method for the compressible Navier-Stokes equations

Daniel T. Graves, Phillip Colella, David Modiano, Jeffrey Johnson, Bjorn Sjogreen and Xinfeng GaO

Second-order accuracy of volume-of-fluid interface reconstruction algorithms

II: An improved constraint on the cell size

\section{Elbridge Gerry Puckett}

Computational models of material interfaces for the study of extracorporeal shock wave therapy

Kirsten Fagnan, Randall J. LeVeque and Thomas J. Matula 